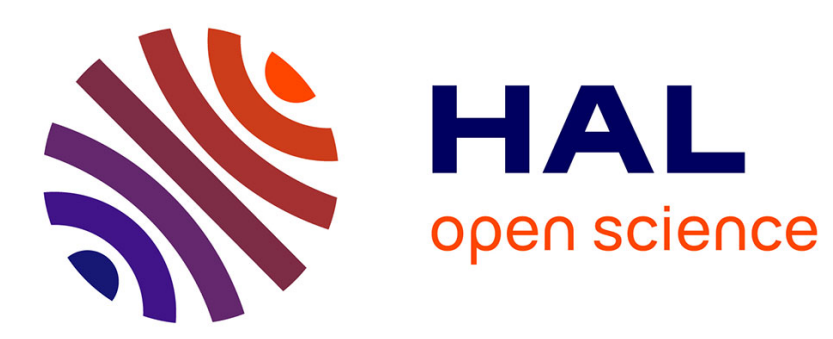

\title{
Models-as-Usual for Unusual Risks? On the Value of Catastrophic Climate Change
}

\author{
Antoine Bommier, Bruno Lanz, Stéphane Zuber
}

\section{To cite this version:}

Antoine Bommier, Bruno Lanz, Stéphane Zuber. Models-as-Usual for Unusual Risks? On the Value of Catastrophic Climate Change. 2014. halshs-00973491

\section{HAL Id: halshs-00973491 \\ https://shs.hal.science/halshs-00973491}

Submitted on 4 Apr 2014

HAL is a multi-disciplinary open access archive for the deposit and dissemination of scientific research documents, whether they are published or not. The documents may come from teaching and research institutions in France or abroad, or from public or private research centers.
L'archive ouverte pluridisciplinaire HAL, est destinée au dépôt et à la diffusion de documents scientifiques de niveau recherche, publiés ou non, émanant des établissements d'enseignement et de recherche français ou étrangers, des laboratoires publics ou privés. 


\section{Documents de Travail du Centre d'Economie de la Sorbonne}

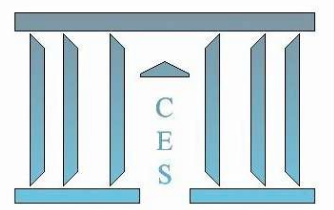

\section{Models-as-Usual for Unusual Risks? On the Value of Catastrophic Climate Change}

Antoine BoMmIER, Bruno LANZ, Stéphane ZUBER

2014.17

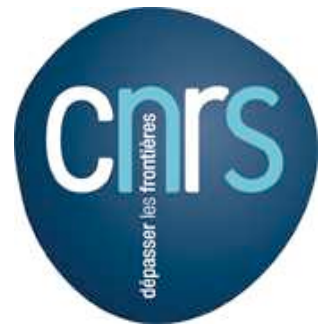




\title{
Models-as-Usual for Unusual Risks? On the Value of Catastrophic Climate Change*
}

\author{
Antoine Bommier ${ }^{\mathrm{a}}$ \\ ETH Zürich
}

\author{
Bruno Lanz ${ }^{\mathrm{b}}$ \\ Graduate Institute Geneva
}

\author{
Stéphane Zuber ${ }^{\mathrm{c}}$ \\ Paris School of \\ Economics - CNRS
}

March 2014

\begin{abstract}
We study the role of alternative intertemporal preference representations in a model of economic growth, stock pollutant and endogenous risk of catastrophic collapse. We contrast the traditional "discounted utility" model, which assumes risk neutrality with respect to intertemporal utility, with a multiplicative choice model that displays risk aversion in that dimension. First, we show that both representations of preferences can rationalize the same "business as usual" economy for a given interest rate and no pollution externality. Second, once we introduce a collapse risk whose hazard rate is a function of the pollution stock, multiplicative preferences recommend a much more stringent policy response. An illustration in the context of climate change indicates that switching to the multiplicative preference representation has a similar effect, in terms of policy recommendations, as scaling up the schedule of the hazard rate by a factor of 100 .

Résumé - Nous étudions les implications de différentes représentations des préférences intertemporelles dans un modèle de croissance avec un stock de pollution et un risque catastrophique endogène. Nous comparons le modèle traditionnel de l'utilité escompté, qui est neutre par rapport au risque sur l'utilité intertemporelle, avec un modèle de choix multiplicatif qui présente une aversion pour ce risque. Nous montrons tout d'abord que les deux modèles peuvent rationaliser la même économie de « statu quo » sans externalité de pollution, pour un niveau donné du taux d'intérêt. Lorsque nous introduisons un risque catastrophique dont la probabilité dépend du stock de pollution, nous montrons en revanche que le modèle de choix multiplicatif recommande une politique climatique plus forte. Nous proposons une illustration numérique avec un modèle de changement climatique qui indique que le passage au modèle multiplicatif a un impact qualitativement semblable (en terme de politique climatique) à une multiplication par $100 \mathrm{du}$ risque instantané de transition vers un état post-catastrophe climatique.
\end{abstract}

Keywords: Environmental policy; Climate change; Catastrophic risks; Risk aversion; Discounting.

Mots clés : Politique environnementale ; Changement climatique ; Risques catastrophiques; Aversion pour le risque ; Escompte.

JEL Classification numbers: D63, D81, D99, Q53, Q54.

\footnotetext{
*We thank Marc Fleurbaey, Reyer Gerlagh, Rick van der Ploeg, Tom Rutherford, Tim Swanson, and participants in the 2013 Annual Meeting of the European Economic Association, the PIIRS workshop on the Ethics of Risk and Climate Change in Princeton, the CRAG-IRGC symposium in Lausanne, the workshop on natural resources organized by NRU-HSE and EUSP in Saint Petersburg, and Applied Economics seminar at Paris School of Economics for helpful discussions and comments. Financial support from Swiss Re is gratefully acknowledged. Any remaining errors are ours.

${ }^{a}$ Chair for Integrative Risk Management and Economics, ETH Zürich, Switzerland. E-mail: abommier@ethz.ch. Tel: +1 6092583506.

${ }^{\mathrm{b}}$ Center for International Environmental Studies, Graduate Institute Geneva, Switzerland; Chair for Integrative Risk Management and Economics, ETH Zürich, Switzerland. E-mail: bruno.lanz@graduateinstitute.ch.

${ }^{\mathrm{c}}$ Centre d'Economie de la Sorbonne, Maison des Sciences Economiques, 106-112 Boulevard de l'Hopital, 75647 Paris Cedex 13, France. E-mail: stephane.zuber@univ-paris1.fr. Tel.: +33144078917.
} 


\section{Introduction}

A substantial part of the economic literature on climate change discusses climate policy as an intertemporal trade-off, where the costs of an early intervention are compared with the costs of later measures. Such an approach is for example the baseline of the prominent contributions by Stern (2007) and Nordhaus (2008). This line of literature typically presumes that anthropogenic climate change is gradual and reversible, and that the world, as we know it, will exist for ever or, at least, that its existence may not be endangered by today's action (or inaction). However, recent literature on climate change reveals the very large uncertainty about how greenhouse gases (GHG) could affect global equilibrium temperatures, particularly in the upper-end of the distribution (Roe and Baker, 2007; Allen and Frame, 2007), and shows an increasing concern for the risk of abrupt and irreversible changes in the climate system (e.g. Alley et al., 2003; Lenton et al., 2008; Kriegler et al., 2009). ${ }^{1}$ There is then the possibility that preventive measures that could be taken today may no longer be available in the future, as a "collapse" might occur in between. The relevant trade-off is no longer that of present consumption versus future consumption, but that of consumption versus a risk of catastrophic climate change (Weitzman, 2009).

This paper considers a setting where a stock of emissions (GHG) increases the hazard risk of a catastrophic collapse. We define a catastrophic collapse as an irreversible regime shift, with post-collapse welfare independent of current actions. Such a setting has been studied in several contributions, starting with Cropper (1976) and including Clarke and Reed (1994), Tsur and Zemel (1996, 1998), Gjerde et al. (1999), and Karp and Tsur (2011) among others. The most pessimistic view would be to interpret such a catastrophic collapse as the end of humankind as we know it, but it can also consist in falling back in a kind subsistence level. What matters for the analysis is that trajectories after the regime shift are exogenous, so that it is not possible to invest towards post-collapse welfare. ${ }^{2}$ Such a

\footnotetext{
${ }^{1}$ The study by Lenton et al. (2008) characterizes potentially abrupt and long-lasting changes for some major elements of the climate system, such as the weakening or shut down of the North Atlantic thermohaline circulation, the melting of the Greenland and the West Antarctic ice sheets, the die-back of the Amazon rainforest and the increasing frequency and amplitude of El Niño-Southern Oscillation. Such low-probability high-impact events pose major challenges to decision-makers, because of the large uncertainty about the associated hazard rates, about the relation of these events to human emissions, and about their impact on humankind.

${ }^{2}$ An alternative approach is to consider the risk of "tipping points", where the realization of the risk affects capital stock through a damage function, but where the fundamental structure of the system is not affected
} 
setting is a natural framework to consider a concept akin to the value of a statistical life at the individual level, represented by the marginal rate of substitution between current consumption and the hazard risk of a regime shift.

Our main objective is to highlight the importance of intertemporal preference representation when considering the trade-off between consumption and catastrophic risk reduction. In the presence of a collapse risk, the time at which the regime shift occurs is a random variable that generates a risk on intertemporal utility. For this kind of problem, relying on the standard expected discounted utility model is a very peculiar choice, as it involves an assumption of risk neutrality with respect to intertemporal utility (Bommier, 2006). One may however think that for a given expected time of collapse, a social planner should prefer to avoid the possibility of a catastrophic collapse occurring in the very short term, as it would result in a very low level of intertemporal utility. In other words, one may want to account for risk aversion with respect to intertemporal utility. In addition, the standard expected discounted utility model makes it impossible to separate intertemporal elasticity of substitution and risk aversion (Epstein and Zin, 1989). As was suggested by Epstein and Zin (1989), a way to achieve this separation involves using the framework of dynamic choice theory of Kreps and Porteus (1978) and allowing for preference for the timing of resolution of uncertainty. This route was followed by many contributions in finance, but also by several works on climate related issues (e.g. Lemoine and Traeger (2013), Cai et al. (2013)). Working in that direction, however, involves allowing for non monotonicity of preferences which can lead to unfortunate conclusions (Chew and Epstein (1990) Bommier and LeGrand (2013)). Instead, this paper will use multiplicative preferences, that separate risk aversion and elasticity of substitution, without having any of these shortcomings.

Multiplicative preferences fit in the expected utility framework and therefore do not introduce preferences for the timing. Just like in the standard expected discounted utility model, information is assumed to have no direct contribution on utility, and is positively valued by the agent only to the extent that it can be used to adjust his/her strategy. The key difference between multiplicative preferences and the standard discounted utility model

and thus continues afterward (see Keller et al., 2004; Lemoine and Traeger, 2013; Cai et al., 2013). In this setting, it is possible to invest towards welfare enjoyed after the risk is realized (see Tsur and Withagen, 2013). In contrast, we consider the case where welfare after the collapse is independent of current actions, so that we rather consider a collapse of civilization or the natural world as we know these concepts (Weitzman, 2009). 
is that instantaneous utilities are aggregated in a multiplicative way, rather than in an additive one. This introduces concavity in the intertemporal utility index, and thus risk aversion over intertemporal welfare, independently from the curvature of the instantaneous utility function. Such preferences, although much less widespread than additive ones, were used to address a number of issues, including portfolio choices (Pye, 1973), precautionary savings (van der Ploeg, 1993), and the equity premium (Ahn, 1989). In a social choice setting, Bommier and Zuber (2008) have provided an axiomatic characterization of a multiplicative model fulfilling the assumption of preference stationarity. An important feature of stationary multiplicative models is that they rule out pure time preferences. Nevertheless time discounting arises from the combination of a risk of collapse and risk aversion with respect to intertemporal utility, thus reconciling time discounting with an equal treatment of generations.

As will be shown in the paper the standard discounted utility model and the multiplicative model can actually yield the same social discount rate when consumption and the risk of collapse are constant. The present paper exploits this result to compare the two models. In particular, using a traditional revealed preference approach, the steady state of an economy with no endogenous climatic risks - which we will refer to as a "business as usual" (BAU) economy - is taken as a common calibration target. Thus instead of discussing how model's predictions are impacted when changing a given parameter, or introducing another behavioral trait, we will use different specifications to rationalize the same economy with exogenous collapse risk, and discuss the divergence in policy recommendation when we introduce a hazard rate that depends on the stock of pollution.

Our results indicate that although additive and multiplicative preferences can reproduce the same steady state equilibrium of a BAU economy, they end up providing divergent conclusions when the collapse risk is viewed as endogenous. The multiplicative model typically generates a much higher social value of catastrophic risk reduction, as measured by the inverse of the marginal rate of substitution between consumption and hazard risk of collapse at a given date. The practical relevance of our results is illustrated through a set of numerical simulations. As a first step we calibrate the parameters of our model to approximate BAU trajectories of the dynamic integrated model of climate and the economy (DICE) model by Nordhaus (2008), and show that these are observationally equivalent under additive and 
multiplicative preference representations. We then introduce an endogenous collapse risk represented by a hazard rate function. We find that the multiplicative model suggests a significantly higher willingness to reduce the risk of collapse, and thus a more rapid introduction of emissions control. Thus, although the choice between models might be considered as purely theoretical with little practical implication in a BAU economy (i.e. when ignoring climate risks), we show that it is a major driver of the policy response to the existence of catastrophic climate risks.

The remainder of the paper is organized as follows. In Section 2 we first present the technological setting. Section 3 is about the social planner's preferences, as we introduce both additive and multiplicative choice models. Section 4 exposes the first order conditions that characterize optimal trajectories when working with these models, and also characterize the steady states. Section 5 reports a quantitative illustration, contrasting optimal paths for additive and multiplicative models under specific risk profiles. Section 6 concludes.

\section{The economy}

Time is assumed to be continuous and denoted by the letters $t, \tau$ or $T{ }^{3}$ To simplify notation, for any function $x(\cdot)$ that depends on time, we denote its value at time $t$ by $x_{t}$ (and not by $x(t))$. The steady state value of a variable $x_{t}$ is denoted as $x^{*}$, and its entire trajectory over time is $x$. Consistent with this notation we will denote the value of the functional $G$ applied to the profile $x$ by $G(x)$ and the value of the function $g$ taken at the point $x_{t}$ by $g\left(x_{t}\right)$. For any function of time $x_{t}$, the notation $\dot{x}_{t}$ will refer to the derivative of $x$ with respect to $t$, i.e. $\dot{x}_{t}=\frac{d}{d t} x_{t}$.

Consider an economy where output is associated with a flow of emissions, which we interpret as GHG emissions. Potential output at time $t$ is denoted by $y_{t}$. It is related to physical capital at time $k_{t}$ through $y_{t}=f\left(k_{t}\right)$. The production function $f$ is assumed to be increasing and concave. Like in Stockey (1998), emissions are caused by production depending on an index of the technology $z_{t}$, with $0 \leq z_{t} \leq 1$. Actual output at time $t$ is $\bar{y}_{t}=z_{t} f\left(k_{t}\right)$. The ratio of GHG emission $e_{t}$ on potential output is given by the differentiable, increasing,

\footnotetext{
${ }^{3}$ Working in continuous time allows us to obtain simpler analytical expressions, and we thus use it for the first sections of the paper. For the quantitative part later on, we will formulate the problem in discrete time. Moving from continuous time to discrete time does not affect the qualitative properties of the problem.
} 
convex function $\varphi\left(z_{t}\right)=\frac{e_{t}}{f\left(k_{t}\right)}$. GHG concentration in the atmosphere $M_{t}$ develops as:

$$
\dot{M}_{t}=e_{t}-\psi\left(M_{t}-\bar{M}\right)=\varphi\left(z_{t}\right) f\left(k_{t}\right)-\psi\left(M_{t}-\bar{M}\right)
$$

where $\psi$ is a positive real number (the natural rate of absorption), and $\bar{M}$ is an equilibrium value for GHG concentration in the absence of anthropogenic GHG emissions.

We assume the existence of a risk of catastrophic collapse that would move the economy to a state where consumption and production trajectories are exogenous (i.e. independent of pre-collapse actions). Intuitively, the nature of this shift is so unpredictable that it is impossible to readily invest for post-collapse welfare. Following Clarke and Reed (1994), we assume that the likelihood of such a collapse is a function of the pollution stock. Formally, we write the hazard rate of a regime shift as:

$$
\mu_{t}=\mu\left(M_{t}\right)
$$

where $\mu$ is an increasing function. Furthermore, we will assume that $\mu(0)=\mu_{0}>0$, with $\mu_{0}$ small, so that the hazard rate of collapse also captures exogenous natural events. Note that we do not consider direct damages associated with increased emissions, either through damages reducing consumption or as an independent element of people welfare. This would solely increase incentives to reduce emissions and distract us from the main point of the paper.

The last aspect of the economy is a simple growth model with only one productive sector. Each period, actual output $\bar{y}_{t}$ can be either consumed $c_{t}$ or invested to accumulate physical capital. The motion equation for capital is:

$$
\dot{k}_{t}=z_{t} f\left(k_{t}\right)-\delta k_{t}-c_{t}
$$

where $\delta$ is the instantaneous rate of capital depreciation. The planner's problem involves choosing the optimal trajectories for $c_{t}$ and $z_{t}$, his objective being discussed in the next section. 


\section{Social preferences}

We consider a social planner who deems that welfare at time $t$ exclusively depends on consumption at time $t$. The planner is aware of the possibility of a regime shift, and that he cannot have any influence on what happens after such a shift. Since the regime shift does not occur deterministically, the planner needs to compare random trajectories. We assume that he is an expected utility maximizer, in the general sense that the utility function is the expected value of intertemporal utilities enjoyed under alternative state of the worlds. In our context, the planner considers the intertemporal utility associated with all possible collapse dates, and compute the expected value using the probability distribution of collapse dates. It is worth emphasizing that assuming expected utility does not involve making assumptions regarding how consumption is aggregated over time to provide an intertemporal utility. The most common approach consists in assuming that intertemporal utility equals the sum of instantaneous utilities, but other aggregation procedures (e.g. a multiplicative aggregation) are of course possible.

Formally, the time at which the world will experience a regime shift is viewed ex-ante as random. Therefore, the planner assesses an infinitely long consumption plan $c$, which may be interrupted by a regime shift. We will denote the utility the planner associates to the case where the consumption profile $c$ is interrupted by a regime shift at time $t$ by $U(c, t)$. The planner will choose $c$ in order to maximize his expected utility, denoted $W(c)$, which is simply the expected value of $U(c, t)$ given $g_{t}$, the distribution function of the random variable $t$ :

$$
W(c)=\int_{0}^{+\infty} U(c, t) g_{t} d t
$$

Further denote the probability that the world will still be in the usual regime at time $t$

(the survival probability) by $s_{t}$. We know from survival analysis that $\mu_{t}=\frac{g_{t}}{s_{t}}=-\frac{\dot{s}_{t}}{s_{t}}$. In consequence, $s_{t}=\exp \left(-\int_{0}^{t} \mu_{\tau} d \tau\right)$ and

$$
g_{t}=\mu_{t} \exp \left(-\int_{0}^{t} \mu_{\tau} d \tau\right)=\mu\left(M_{t}\right) \exp \left(-\int_{0}^{t} \mu\left(M_{\tau}\right) d \tau\right)
$$


We can thus re-express the social expected utility (2) as:

$$
W(c)=\int_{0}^{+\infty} U(c, t) \mu\left(M_{t}\right) \exp \left(-\int_{0}^{t} \mu\left(M_{\tau}\right) d \tau\right) d t
$$

Hence the social welfare function is a function of the consumption path as well as the hazard rate associated with the trajectory of the pollution stock.

We now impose further structure on the planner's preferences. First, we assume that preferences are stationary in the sense of Koopmans (1960). This reflects the view that preferences regarding the future should be independent of the past and of the calendar date, and ensures that the ensuing plans are time consistent. Second, we assume that preferences are weakly separable in the sense that their restriction to deterministic consumption paths fulfills the separability assumption described in Gorman (1968). In other words, in absence of uncertainty, the trade off between welfare at two different moments in time is independent from what happens in other periods.

As is shown in Bommier (2012), these standard assumptions imply that the utility index, whose expectation is maximized by the planner, must have either an additive or a multiplicative structure. More specifically, assuming that exogenous post-collapse welfare is equivalent to the welfare associated with consuming $\underline{c}$ forever, and normalizing (without loss of generality) the utility function $u(\cdot)$ so that $u(\underline{c})=0$, we must have:

$$
\begin{gathered}
U(c, t)=\int_{0}^{t} e^{-\theta \tau} u\left(c_{\tau}\right) d \tau \quad \text { in the additive case, } \\
U(c, t)=\frac{1-\exp \left\{-\varepsilon \int_{0}^{t} u\left(c_{\tau}\right) d \tau\right\}}{\varepsilon} \quad \text { in the multiplicative case, }
\end{gathered}
$$

where $\theta \geq 0$ is the pure rate of time preference in the additive model and $\varepsilon \geq 0$ measures the degree of absolute risk aversion with respect to intertemporal utility in the multiplicative model (i.e. an increase in $\varepsilon$ increases risk aversion in the sense of Kihlstrom and Mirman (1974)). In the remainder of the paper, we call $\varepsilon$ the temporal risk aversion parameter.

The additive model (5) is the one that has been used by most of the literature. The behavior of the society is governed by two main features: pure time preference which is embodied in the parameter $\theta$, and the intertemporal elasticity of substitution embodied 
in the curvature of function $u$. With the multiplicative model, intertemporal elasticity of substitution is still embodied in the curvature of the function $u$. Time preference is set to zero and another parameter $\varepsilon$ is introduced. This parameter has no impact on preferences over deterministic consumption paths. It is however key for the evaluation of random prospects. Actually going from a smaller to a larger value of $\varepsilon$ involves taking a concave transformation of the (lifetime) utility index, which in expected utility theory means increasing risk aversion (Kihlstrom and Mirman, 1974). Thus, with the multiplicative model, one element (the function $u$ ) determines preferences in absence of uncertainty, and another one (the scalar $\varepsilon)$ determines the degree of risk aversion, with no impact on preferences over deterministic consumption paths. The desired separation between intertemporal elasticity of substitution and risk aversion is thus achieved, while retaining the same number of degrees of freedom as in the standard discounted utility model.

The additive and multiplicative models are both extensions of the case where utility would be given by $U(c, t)=\int_{0}^{t} u\left(c_{\tau}\right) d \tau$. This specification is indeed obtained when $\theta=0$ in the additive case and when $\varepsilon=0$ in the multiplicative case. In the expected utility framework, it is impossible to combine pure time preference and risk aversion with respect to intertemporal utility without giving up the assumption of preference stationarity. Stationary preferences exhibiting both pure time preferences and risk aversion with respect to intertemporal utility may be obtained by leaving the domain of expected utility theory, as in the "risk-sensitive preferences" model introduced by Hansen and Sargent (1995) and axiomatized in Bommier and LeGrand (2013). In fact, additive and multiplicative models are just two polar cases in the class of risk-sensitive preferences. Thus, our results could be extended to a continuum of models in which $\theta>0$ and $\varepsilon>0$. Focusing on additive and multiplicative specifications is however sufficient, and the simplest way to provide insights about the importance of the representation of intertemporal preferences. ${ }^{4}$

Normative and ethical aspects of additive and multiplicative preferences have been discussed in Bommier and Zuber (2008). In short, pure time preferences in the additive model imply that different generations are given different utility weights as a consequence of their years or birth. This has often been criticized for being contrary to intergenerational equity.

\footnotetext{
${ }^{4}$ One technical consideration worth mentioning is that the integral in Equation (2) does not necessarily converge. For the problem at hand however, and assuming that $\theta$ and $\varepsilon$ are non-negative, this issue can be avoided by making the assumption that consumption is bounded.
} 
Multiplicative preferences rule out pure time preferences but make it possible for the the planner to exhibit risk aversion with respect to intertemporal utility. This may lead the social planner to choose policies that do not optimize individual expected welfare in order to reduce the aggregate risk (Bommier and Zuber, 2008). There is a debate about such ethical implications (Manski and Tetenov, 2007; Fleurbaey, 2010), and we do not suggest that one model is more appropriate than the other. Rather, our aim is to highlight implications of alternative preferences towards social risk in the context of climate change policy.

For both additive and multiplicative preferences, we can apply integration by part on equation (4) to obtain:

$$
W(c)=\left[-U(c, t) \exp \left(-\int_{0}^{t} \mu\left(M_{\tau}\right) d \tau\right)\right]_{0}^{+\infty}+\int_{0}^{+\infty} \frac{d U(c, t)}{d t} \exp \left(-\int_{0}^{t} \mu\left(M_{\tau}\right) d \tau\right) d t
$$

The first term of the right hand side is zero, and the second one can be simplified by introducing a function $v\left(\mu_{t}, c_{t}\right)$ from $\mathbb{R} \times \mathbb{R}$ into $\mathbb{R}$ given by:

$$
\begin{gathered}
v\left(\mu_{t}, c_{t}\right)=\theta+\mu_{t} \quad \text { in the additive case, } \\
v\left(\mu_{t}, c_{t}\right)=\mu_{t}+\varepsilon u\left(c_{t}\right) \quad \text { in the multiplicative case, }
\end{gathered}
$$

We can now write equation (7) as:

$$
W(c)=\int_{0}^{+\infty} \exp \left(-\int_{0}^{t} v\left(\mu\left(M_{\tau}\right), c_{\tau}\right) d \tau\right) u\left(c_{t}\right) d t
$$

Using equation (10) is convenient, as the difference between both models is restricted to the function $v\left(\mu_{t}, c_{t}\right)$, defined in (8) and (9).

For notational purposes, we write the continuation utility $\mathcal{U}(c, T)$ as the future remaining expected utility discounted at time $T$ :

$$
\mathcal{U}(c, T)=\int_{T}^{+\infty} \exp \left(-\int_{T}^{t} v\left(\mu\left(M_{\tau}\right), c_{\tau}\right) d \tau\right) u\left(c_{t}\right) d t
$$

We shall now define two important concepts. 
Definition 1 The social discount rate is:

$$
\rho(c, T)=-\left.\frac{d}{d T}\left(\log \frac{\partial W(c)}{\partial c_{T}}\right)\right|_{\dot{c}_{T}=0}
$$

The discount rate measures how rapidly the marginal utility of consumption is declining with time, controlling for variations in consumption. ${ }^{5}$ A similar definition can for example be found in Epstein (1987).

The second concept quantifies the willingness to sacrifice current consumption to lower the hazard risk of a regime shift. It is similar to what Weitzman (2009) refers to as "the value of statistical civilization", or "VSL-like parameter".

Definition 2 The social value of catastrophic risk reduction is:

$$
\mathcal{V}(c, T)=-\left(\frac{\partial W(c)}{\partial \mu_{T}}\right) /\left(\frac{\partial W(c)}{\partial c_{T}}\right)
$$

This social value of catastrophic risk reduction is therefore simply the opposite of the marginal rate of substitution between consumption at time $T$ and hazard risk of regime shift at time $t$. In the parallel literature on individual choice with endogenous mortality (death being a particular regime shift), this is called the value of a statistical life.

The following proposition provides expressions for the social discount rate and for the social value of catastrophic risk reduction for the additive and multiplicative cases:

\section{Proposition 1}

(a) In the additive case, the social discount rate and the social value of catastrophic risk reduction are given by the expressions $\rho(c, T)=\mu\left(M_{T}\right)+\theta$ and $\mathcal{V}(c, T)=\frac{\mathcal{U}(c, T)}{u^{\prime}\left(c_{T}\right)}$.

(b) In the multiplicative case, the social discount rate and the social value of catastrophic risk reduction are given by the expressions $\rho(c, T)=\frac{\mu\left(M_{T}\right)}{1-\varepsilon \mathcal{U}(c, T)}$ and $\mathcal{V}(c, T)=\frac{\mathcal{U}(c, T)}{u^{\prime}\left(c_{t}\right)(1-\varepsilon \mathcal{U}(c, T))}$.

Proof. See Appendix A.

In the additive model, the discount rate is just the sum of the rate of pure time preferences and of the hazard risk of regime shift, as is well known since Yaari (1965). In particular

\footnotetext{
${ }^{5}$ As we work in continuous time, the derivatives $\frac{\partial W(c)}{\partial c_{T}}$ is to be understood as a Volterra derivative.
} 
the rate of time discounting is independent of consumption. In the multiplicative model, the discount rate involves the continuation utility, which depends on future consumption, and future hazard risk. This implies that changing the parameters of the multiplicative model has a non-trivial impact on the discount rate as the continuation utility function may depend on these parameters. Along a trajectory where the hazard rate of a regime shift and consumption are constant over time, however, the discount rate for the multiplicative model is constant and equal to $\rho\left(c^{*}, T\right)=\mu+\varepsilon u\left(c^{*}\right)$. This has two implications. First, the discount rate is increasing in $\varepsilon$. Intuitively, if a regime shift occurs, later generations will not be able to benefit from foregone consumption by early generations, which leads a risk averse planner to put more weight on the welfare of early generations. Second, and more importantly, both models will exhibit the same discount rate when $\theta=\varepsilon u\left(c^{*}\right)$. Thus although both models rely on different fundamental assumption regarding pure time preferences and risk aversion, in presence of a collapse risk they may end up generating an identical degree of time discounting. This explains why we will find that both the additive and the multiplicative models may rationalize the same equilibrium steady state in a BAU economy (see the discussion following Proposition 2).

As we have mentioned in the introduction, this ability of both models to generate identical discount rates makes it possible to compare these models with a revealed preference approach. We will therefore not consider the discussion of what happens when increasing $\theta$ in the additive model, or when changing $\varepsilon$ in the multiplicative model. Instead we will compare different specifications which, for a given exogenous collapse risk, would exhibit identical discount rates. As will be shown in Sections 5.1 and 5.2, these are specifications that generate identical steady states and very similar transitory paths in BAU economies.

The second part of Proposition 1 shows that the additive and multiplicative models provide very different expressions for the social value of catastrophic risk reduction. In both cases this value depends positively on the continuation utility (thus on future consumption), as it increases the cost of a regime shift, and thus the willingness to pay to lower its risk. In the additive case, continuation utility is a decreasing function of the rate of time preference. Thus a higher preference for the present imply a lower willingness to pay for risk reduction. In the multiplicative case the role of temporal risk aversion is less obvious, as it combines two effects. On the one hand, a greater $\varepsilon$ implies a higher aversion to the risk of regime shift, 
thus increasing the willingness to pay for risk reduction. On the other hand, in presence of a collapse risk, a greater $\varepsilon$ increases the value of immediate consumption through the discount rate, which lowers the willingness to pay for risk reduction. The overall effect is ambiguous, which is in line with findings on the role of risk aversion on preventive measures (Dionne and Eeckhoudt, 1985; Jullien et al., 1999).

In order to gain further intuition about the implications of additive and multiplicative preferences in the presence of an endogenous collapse risk, one can observe that in the additive case:

$$
\mathcal{V}(c, T)=\frac{\rho(c, T) \mathcal{U}(c, T)}{u^{\prime}\left(c_{T}\right)} \frac{1}{\mu_{t}+\theta}
$$

while in the multiplicative case:

$$
\mathcal{V}(c, T)=\frac{\rho(c, T) \mathcal{U}(c, T)}{u^{\prime}\left(c_{T}\right)} \frac{1}{\mu_{t}}
$$

If consumption and the hazard rate of regime shift were to remain constant over time, then in both models, one would have $\rho\left(c^{*}, T\right) \mathcal{U}\left(c^{*}, T\right)=u\left(c^{*}\right)$. Further considering the relevant case where both models imply the same discount rate, the value of catastrophic risk reduction would be $\left(1+\frac{\theta}{\mu}\right)$ larger in the multiplicative model than in the additive one. Predictions of both models would then strongly diverge when the pure rate of time preference in the additive model is large compared to the hazard risk.

\section{Optimal paths and steady states}

\subsection{Business as usual}

We first study the problem of choosing an optimal consumption plan when the hazard risk of collapse is constant and exogenous. We label this problem the BAU problem because the planner does not account for the effect of emissions on the risk of a catastrophic event. Our objective is to highlight that additive and multiplicative preferences may yield observationally equivalent equilibria in problems that focus on intertemporal consumption trade-offs. This contrasts with a setting in which the planner accounts for the effects of current decisions on the hazard of a catastrophic collapse, which we discuss next (Section 4.2).

Consider a social planner who faces a hazard risk $\mu_{t}=\mu_{0}$ in all periods. His objective, 
which defines the BAU problem, is:

$$
\begin{array}{ll}
\max _{(c, z)} \quad & W(c)=\int_{0}^{+\infty} \exp \left(-\int_{0}^{t} v\left(\mu\left(M_{\tau}\right), c_{\tau}\right) d \tau\right) u\left(c_{t}\right) d t \\
\text { s.t. } \quad & \dot{k}_{t}=z_{t} f\left(k_{t}\right)-\delta k_{t}-c_{t} \\
& \mu(M)=\mu_{0}, \quad \forall M \in \mathbb{R}
\end{array}
$$

with $v\left(\mu_{t}, c_{t}\right)=\theta+\mu_{0}$ in the additive case and $v\left(\mu_{t}, c_{t}\right)=\mu_{0}+\varepsilon u\left(c_{t}\right)$ in the multiplicative case.

Because the stock of pollution does not have a direct effect on welfare and the impact of pollution on the regime shift is overlooked by the planner, there will be no emission control. Hence at the optimum we have $z_{t}=1$ for all $t \in \mathbb{R}$. In this situation, a steady state corresponds to an optimal path such that $\dot{c}_{t}=\dot{k}_{t}=0{ }^{6}$

\section{Proposition 2}

(a) If an interior solution of Problem (13) exists, then it is characterized by the following equations:

$$
\begin{aligned}
& \dot{c}_{t}=-\frac{u^{\prime}\left(c_{t}\right)}{u^{\prime \prime}\left(c_{t}\right)}\left(f^{\prime}\left(k_{t}\right)-\delta-\rho(c, t)\right) \\
& \dot{k}_{t}=f\left(k_{t}\right)-c_{t}
\end{aligned}
$$

(b) If a steady state exists for an interior solution of Problem (13), then:

(i) In the additive case, it is characterized by the following equations:

$$
\begin{aligned}
\mu_{0}+\theta & =f^{\prime}\left(k^{*}\right)-\delta \\
f\left(k^{*}\right) & =c^{*}
\end{aligned}
$$

(ii) In the multiplicative case, it is characterized by the following equations:

$$
\begin{aligned}
\mu_{0}+\varepsilon u\left(c^{*}\right) & =f^{\prime}\left(k^{*}\right)-\delta \\
f\left(k^{*}\right) & =c^{*}
\end{aligned}
$$

${ }^{6}$ We know that $\dot{z}_{t}=0$. There is no reason to assume that $\dot{M}_{t}=0$ because $M_{t}$ does not impact welfare and is therefore irrelevant for the BAU problem. 
Proof. See Appendix B.

The optimal dynamics described in part (a) of Proposition 2 is the familiar optimal growth trajectory in a one-sector model. Equation (14) is the Euler equation describing the evolution of consumption as a function of the difference between the interest rate on capital investments and the social discount rate. Equation (14) holds for both the additive and multiplicative models, but the expression of the discount rate $\rho(c, t)$ depends on the specification of preferences (Proposition 1). Equation (15) is the standard motion equation for the state variable.

A noteworthy feature of Proposition 2 is the similarity of the additive and multiplicative steady state equilibria in the BAU problem, displayed in part (b) of the Proposition. For any $\theta$ it is possible to find an $\epsilon$ such that the additive and multiplicative models yield the same interest rate in the long run, and hence the same equilibrium consumption and physical capital. In particular, it suffices to define $\varepsilon=\theta / u\left(c^{*}\right)$ for the steady state consumption and physical capital to be the same in the additive and multiplicative cases.

While the parameters of the models can be calibrated to produce the same BAU steady state equilibrium, short-term dynamics will differ. For such a calibration, our quantitative illustration will however suggest that optimal BAU paths for the additive and multiplicative models are very similar (see Section 5.2 below).

\subsection{Endogenous catastrophic collapse}

We now come to the specific problem studied in this paper, namely the case where the social planner anticipates the effect of his actions on the probability of a regime shift. In that case, the social planner seeks to solve the following dynamic optimization problem:

$$
\begin{array}{ll}
\max _{(c, z)} \quad & W(c)=\int_{0}^{+\infty} \exp \left(-\int_{0}^{t} v\left(\mu\left(M_{\tau}\right), c_{\tau}\right) d \tau\right) u\left(c_{t}\right) d t \\
\text { s.t. } & \dot{k}_{t}=z_{t} f\left(k_{t}\right)-\delta k_{t}-c_{t} \\
& \dot{M}_{t}=\varphi\left(z_{t}\right) f\left(k_{t}\right)-\psi\left(M_{t}-\bar{M}\right)
\end{array}
$$

with $v\left(\mu_{t}, c_{t}\right)=\theta+\mu_{t}$ in the additive case and $v\left(\mu_{t}, c_{t}\right)=\mu_{t}+\varepsilon u\left(c_{t}\right)$ in the multiplicative case. 
Defining $\pi(z)=z-\frac{\varphi(z)}{\varphi^{\prime}(z)}$, the optimal path for an interior solution of Problem (20) is characterized as follows:

\section{Proposition 3}

(a) If an interior solution of Problem (20) exists, it is characterized by the following equations:

$$
\begin{gathered}
\dot{c}_{t}=-\frac{u^{\prime}\left(c_{t}\right)}{u^{\prime \prime}\left(c_{t}\right)}\left(\pi\left(z_{t}\right) f^{\prime}\left(k_{t}\right)-\delta-\rho\left(c_{t}, t\right)\right) \\
\dot{z}_{t}=\frac{\varphi^{\prime}\left(z_{t}\right)}{\varphi^{\prime \prime}\left(z_{t}\right)}\left(\varphi^{\prime}\left(z_{t}\right) \mu^{\prime}\left(M_{t}\right) \mathcal{V}\left(c_{t}, t\right)+\delta-\left\{\psi+\pi\left(z_{t}\right) f^{\prime}\left(k_{t}\right)\right\}\right) \\
\dot{k}_{t}=z_{t} f\left(k_{t}\right)-c_{t} \\
\dot{M}_{t}=\varphi\left(z_{t}\right) f\left(k_{t}\right)-\psi\left(M_{t}-\bar{M}\right)
\end{gathered}
$$

(b) If a steady state exists for an interior solution of Problem (20), then:

(i) In the additive case, it is characterized by the following equations:

$$
\begin{aligned}
\mu\left(M^{*}\right)+\theta & =\pi\left(z^{*}\right) f^{\prime}\left(k^{*}\right)-\delta \\
\pi\left(z^{*}\right) f^{\prime}\left(k^{*}\right)-\delta+\psi & =\frac{u\left(c^{*}\right)}{u^{\prime}\left(c^{*}\right)} \frac{\mu^{\prime}\left(M^{*}\right)}{\mu\left(M^{*}\right)+\theta} \varphi^{\prime}\left(z^{*}\right) \\
z^{*} f\left(k^{*}\right) & =c^{*} \\
\varphi\left(z^{*}\right) f\left(k^{*}\right) & =\psi\left(M^{*}-\bar{M}\right)
\end{aligned}
$$

(ii) In the multiplicative case, it is characterized by the following equations:

$$
\begin{aligned}
\mu\left(M^{*}\right)+\varepsilon u\left(c^{*}\right) & =\pi\left(z^{*}\right) f^{\prime}\left(k^{*}\right)-\delta \\
\pi\left(z^{*}\right) f^{\prime}\left(k^{*}\right)-\delta+\psi & =\frac{u\left(c^{*}\right)}{u^{\prime}\left(c^{*}\right)} \frac{\mu^{\prime}\left(M^{*}\right)}{\mu\left(M^{*}\right)} \varphi^{\prime}\left(z^{*}\right) \\
z^{*} f\left(k^{*}\right) & =c^{*} \\
\varphi\left(z^{*}\right) f\left(k^{*}\right) & =\psi\left(M^{*}-\bar{M}\right)
\end{aligned}
$$

Proof. See Appendix C and Appendix D for the additive and multiplicative cases respectively. 
To understand the optimal dynamics described in part (a) of Proposition 3, first note that Equation (21) is, like before, the Euler equation. It is adapted to the present setting where net interest rate $r_{t}=\pi\left(z_{t}\right) f^{\prime}\left(k_{t}\right)-\delta$ is adjusted to take into account the pollution externality.

Equation (22) is an arbitrage condition on emission control. Assume that you divert money from investments in physical capital to increase emission control (i.e. decrease $z_{t}$ ). The cost is given by the net interest rate $r_{t}$. The benefit is to decrease emissions (in proportion $\left.\varphi^{\prime}\left(z_{t}\right)\right)$, so that the risk decreases through $\mu\left(M_{t}\right)$. The value of this risk reduction is given by $\mathcal{V}\left(c_{t}, t\right)$. Hence the net benefit is $\varphi^{\prime}\left(z_{t}\right) \mu^{\prime}\left(M_{t}\right) \mathcal{V}\left(c_{t}, t\right)-\psi$, where $\psi$ accounts for the reduction in risk that occurs through natural absorption. The arbitrage between costs and benefits governs the evolution of emission control.

A key difference with the BAU problem is that the steady states displayed in part (b) of Proposition 3 are different in the additive and the multiplicative cases when they are calibrated to the same BAU interest rate $\left(\varepsilon=\theta / u\left(c^{*}\right)\right)$. The key difference is in the righthand side of Eq. (26) and (30). The difference tends to vanish when $\theta$ is close to 0 (so that the associated $\varepsilon$ is close to 0 ). Hence, for very low values of the rate of pure time preference (and of the temporal risk aversion parameter), the two models behave in a similar way in the long run. This is consistent with the fact that when $\theta=\varepsilon=0$ the additive and multiplicative preference models are formally identical. However, as soon as we take some distance from this rather extreme case, the models provide very distinct predictions, as pure time preferences and risk aversion with respect to the date of a catastrophic shift have different behavioral implications.

In the additive model, increasing pure time preference has two direct effects. First, it increases the social discount rate (Equation (25)). Second, it decreases the social value of catastrophic risk reduction, which is $\frac{u\left(c^{*}\right)}{u^{\prime}\left(c^{*}\right)\left(\mu\left(M^{*}\right)+\theta\right)}$ in steady state (Equation (26)). From the effect on the social discount rate, the future matters less for higher values of pure time preference, inducing less capital accumulation that can result in less consumption and less pollution. On the contrary, higher values of pure time preference will induce a lower value of catastrophic reduction, and hence laxer environmental policies that can result in more consumption and more pollution.

In the multiplicative model, increasing temporal risk aversion has only one direct effect, 
which is to increase the social discount rate (Equation (29)). A higher value of the temporal risk aversion parameter will therefore tend to decrease consumption but it has no direct impact on the steady state value of catastrophic risk reduction, which is $\frac{u\left(c^{*}\right)}{u^{\prime}\left(c^{*}\right) \mu\left(M^{*}\right)}$. It may still have an indirect impact through its effect on consumption and GHG concentration. It is worth noting that for the same value of $c^{*}$ and $M^{*}$ the social value of catastrophic risk reduction is always higher in the multiplicative model than in the additive one. This suggests that the multiplicative model may be more prone to avoiding catastrophic risks.

To study the magnitude of these effects, we now turn to a numerical version of the model. It will allow us to compare not only the steady state equilibria, but also the optimal dynamics of the two models. We will also investigate the role of two key parameters: the rate of pure time preference $\theta$ (or the associated temporal risk aversion parameter $\varepsilon$ ) and the consumption level $\underline{c}$ equivalent to the post regime shift welfare.

\section{A quantitative illustration}

\subsection{Functional forms, parameters and solution method}

To illustrate quantitatively the implications of the additive and multiplicative models, we proceed in three steps. First, we use our model to approximate BAU trajectories (i.e. no climate externality) for output and the stock of GHG in the DICE model (Nordhaus, 2008). We will do so under the standard additive representation of preferences, taking most functional forms and parameters from Nordhaus (2008), and selecting remaining parameters to fit BAU trajectories. The parameters are summarized in Table 1. Second, we introduce a hazard rate schedule describing the risk of catastrophic collapse as a function of the GHG stock. Third, we specify the social objectives under additive and multiplicative preference representations. This last step will enable us to simulate optimal paths with and without endogeneity in the risk of catastrophic collapse.

Under additive preferences, the BAU problem (13) reduces to a standard optimal growth problem a la Ramsey (see Appendix B). Assuming that potential output is represented by the function $y_{t}=A k_{t}^{\alpha}$, with $\alpha=0.3$ as in Nordhaus (2008), it is straightforward to obtain analytical expressions for steady state quantities. ${ }^{7}$ The total factor productivity (TFP)

\footnotetext{
7 The BAU expressions for the steady state capital stock and consumption given additive preferences are
} 
Table 1: Functional forms and parameters

\begin{tabular}{|c|c|c|}
\hline Parameter & Value & Source \\
\hline \multicolumn{3}{|l|}{ Production technology: $y_{t}=A k_{t}^{\alpha}$} \\
\hline$A$ Total factor productivity & 8.5 & Calibrated \\
\hline$\alpha \quad$ Share of capital & 0.3 & Nordhaus (2008) \\
\hline$k_{0} \quad$ Initial capital stock & 0.99 & Calibrated \\
\hline$\delta \quad$ Rate of depreciation & 0.1 & Nordhaus (2008) \\
\hline \multicolumn{3}{|l|}{ Carbon cycle: $\Delta M_{t}=\kappa z_{t}^{\beta}-\psi\left(M_{t}-\bar{M}\right)$} \\
\hline$\kappa \quad$ Emissions intensity & 0.29 & Calibrated \\
\hline$\beta \quad$ Abatement cost parameter & 2.7 & Nordhaus (2008) \\
\hline$\psi \quad$ Exogenous emissions' decay & 0.005 & Calibrated \\
\hline $\bar{M}$ Pre-industrial stock of emissions & 596.4 & Nordhaus (2008) \\
\hline$M_{0}$ Initial pollution stock & 808.9 & Nordhaus (2008) \\
\hline \multicolumn{3}{|l|}{ Instantaneous utility function: $u(c)=\frac{c^{1-\gamma}-\underline{c}^{1-\gamma}}{1-\gamma}$} \\
\hline$\gamma \quad$ Marginal utility parameter & 2.1 & Calibrated \\
\hline$\underline{c}$ Post-collapse consumption & 1 & - \\
\hline $\bar{\theta} \quad$ Pure rate of time preference (additive model) & 0.015 & Nordhaus (2008) \\
\hline$\varepsilon \quad$ Temporal risk aversion (multiplicative model) & 0.0014 & Calibrated \\
\hline
\end{tabular}

parameter $A$ is then calibrated so that BAU steady state output in our model equals per capita output in 2100 in the DICE model, given the assumed pure rate of time preferences in the additive model (1.5\%, see below) and a depreciation rate of $10 \%$ taken from Nordhaus (2008). The initial capital stock $\left(k_{0}\right)$ is then chosen so that initial output $y_{0}$ equals DICE's per capita output in the initial year (2005).

The DICE model features a detailed representation of the carbon cycle, whereas we only have one equation describing the dynamics of the pollution stock (Equation (1)). Nevertheless, we find that we can approximate GHG dynamics reasonably well. First, the initial pollution stock $M_{0}=808.9 \mathrm{GtC}$ and the pre-industrial $\mathrm{GHG}$ concentration $\bar{M}=596.4 \mathrm{GtC}$ are taken directly from DICE. Second, the natural decay of the stock, measured by $\psi$ in our model, is determined as follows. We simulate the decay of the stock of GHG in the DICE model by switching off emissions from 2005 onwards. We then select the value of $\psi$ that minimizes the sum of squared deviations between the decay trajectory implied by the DICE given by:

$$
k^{*}=\left(\frac{A \alpha}{\delta+\rho}\right)^{\frac{1}{1-\alpha}} \quad \text { and } \quad c^{*}=A\left(k^{*}\right)^{\alpha}-\delta k^{*}
$$


model and that implied by the linear stock dynamics of our model. Third, the function describing emissions per unit of output is taken from the DICE model, i.e. $\varphi\left(z_{t}\right)=\kappa z_{t}^{\beta}$, with $\beta=2.7$. Finally, the parameter $\kappa$, which measures uncontrolled emissions intensity per unit of output, is chosen so as to minimize least squares deviations between the BAU path for the stock of GHG in the DICE model and in our model, given BAU trajectories for $y_{t}$ and the natural decay parameter $\psi$.

The hazard risk of catastrophic collapse is described by the function $\mu(M)=\mu_{0}+$ $\mu_{1}(M-\bar{M})^{\sigma}$. The exogenous hazard parameter is set to $\mu_{0}=10^{-5}$, which implies a survival probability of $90 \%$ after 10,000 years and $40 \%$ after 100,000 years. As the GHG stock increases above $\bar{M}$, the hazard risk increases with parameters $\mu_{1}$ and $\sigma$. We consider two alternative parametrization for the hazard function, illustrated in Figure 1. Under the "low" risk profile, the endogenous part of the hazard risk, $\mu_{1}(M-\bar{M})^{\sigma}$, remains below $\mu_{0}$ when the stock doubles relative to $\bar{M}$, but it increases to $0.01 \%$ when the stock triples. This implies that a tripling of the GHG stock would reduce the survival probability by 2.5 percentage points after 1,000 years. Under the "high" risk profile, the endogenous part of the hazard rate is 100 times larger for the same pollution stock, reaching $1 \%$ for a tripling of the initial stock. Under this profile, a mere doubling of the stock reduces the survival probability by 3 percentage points after only 100 years. ${ }^{8}$

Turning to the representation of social preferences, instantaneous utility is given by $u\left(c_{t}\right)=\frac{c^{1-\gamma}-\underline{c}^{1-\gamma}}{1-\gamma}$. We calibrate $\gamma$ such that the initial saving rate in the BAU is $22 \%$ as in the DICE model. The post-collapse consumption level $\underline{c}$ is set to 1 , which corresponds to an $85 \%$ reduction of the initial consumption level (around $\$ 6,500$ per capita per year). We stress that our aim is not to portray a specific catastrophe linked with climate change. Rather we see this quantitative exercise as a way to illustrate the magnitude of the modeling choices. We will examine the importance of $\underline{c}$ for our results in Section 5.4.

In the additive model, the pure rate of time preference $\theta$ is $1.5 \%$ per year, which is the baseline in the analysis of the DICE model by Nordhaus (2008). As discussed in Section 4.1, we calibrate the temporal risk aversion parameter in the multiplicative model such that

\footnotetext{
${ }^{8}$ This is still a lower bound of the illustrative calculations conducted by Weitzman (2009), as he suggests that there is a one percent probability that climate sensitivity could be above $20^{\circ} \mathrm{C}$. Since climate sensitivity measures the equilibrium change in temperatures following a doubling of GHG concentration, this can be interpreted as the risk associated with a doubling of GHG concentrations. Nevertheless the numbers used in the high risk profile are roughly in the same ballpark.
} 
Figure 1: Pollution stock and hazard risk of collapse

(a) Low risk profile

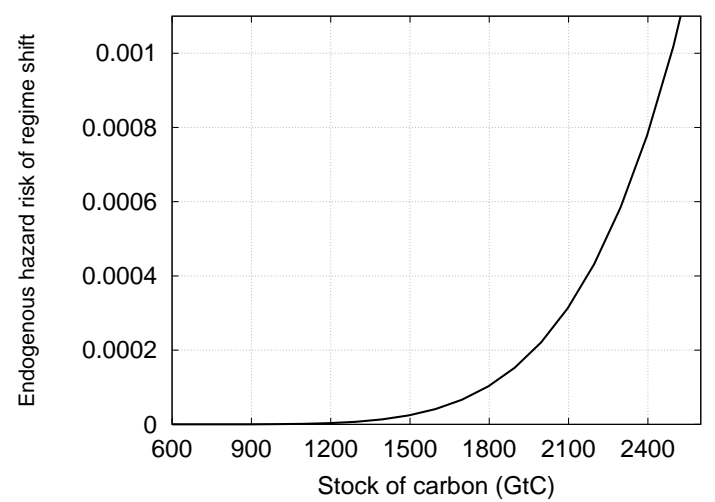

(b) High risk profile

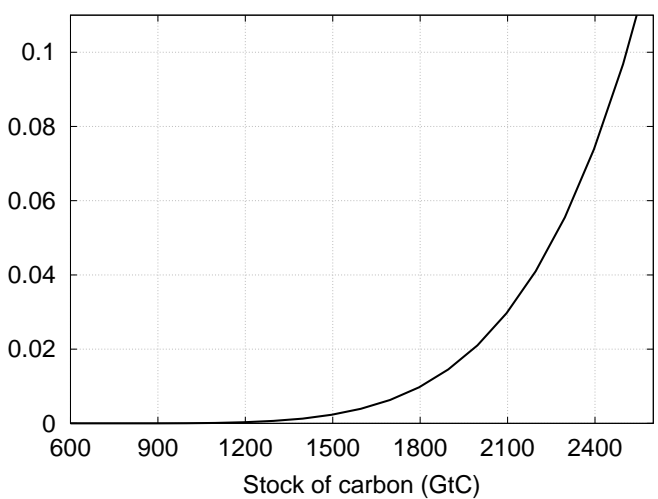

both models predict the same BAU steady state interest rate. This is achieved by setting $\varepsilon=\theta / u\left(c^{*}\right)$, where $c^{*}$ is BAU steady state consumption in the additive model (see footnote 7). We will return to the choice of $\theta$ in Section 5.4.

We solve the numerical versions of problems (13) and (20) as discrete time non-linear programs. Indeed the stochasticity of the problem is summarized in the function $v\left(\mu_{t}, c_{t}\right)$, so that standard tools from mathematical programming can be employed. In an optimization framework however it is not possible to formulate an infinite horizon program, since it would require maximizing a sum with an infinite number of terms subject to an infinite number of constraints. We thus truncate the horizon of the problem to a finite number of periods, and approximate the solution to the infinite horizon problem with a state variable targeting approach (Lau et al., 2002). ${ }^{9}$

\footnotetext{
${ }^{9}$ If the time horizon of the problem is finite, the optimal shadow values of the stock variables are zero in finite time. Hence typically, the solution of the finite horizon problem differs from its infinite horizon counterpart. These terminal effects can be avoided by adding equilibrium conditions targeting the postterminal evolution of the shadow values to be consistent with the infinite horizon solution. This approach involves formulating the problem in the mixed complementarity format, which exploits the equilibrium conditions relating the constraints of the primal problem and their associated multipliers, as defined by the Karush-Kuhn-Tucker conditions. Following Lau et al. (2002), we exploit conditions relating the rate of change of investment to that of output to target the post-terminal value of the capital stock, and similarly we target the post-terminal value of pollution with (the negative of) the rate of change of the technology index $z_{t}$ to that of output. We formulate the system of complementarity relationships in GAMS and solve it with the PATH algorithm (Dirkse and Ferris, 1995).
} 


\subsection{Business as usual and TFP shock}

We start by comparing the BAU paths implied by the additive and multiplicative models. Given our calibration procedure both models admit the same steady state interest rate, and by Proposition 2 the steady state capital stock and consumption level are identical. In the additive case, it is possible to derive closed-form solutions for the steady state values of the capital stock and consumption level (see footnote 7), and this can be used to validate both the calibration procedure and the accuracy of the solution method.

Figure 2 reports the optimal paths for $c_{t}$ and $k_{t}$, as well as the value of their respective analytical steady state. All paths converge to their theoretical values, which demonstrates that both models can rationalize the same BAU economy, but also that we numerically approximate the true steady state with a high precision. Moreover, while the transition paths can potentially differ between the additive and the multiplicative specifications, optimal trajectories are almost identical. We note that the multiplicative model suggests a slightly more rapid convergence towards the steady state. This is because the discount rate is endogenous according to the multiplicative model, and thus changes during the transition.

Figure 2: Business as usual calibration and TFP shock
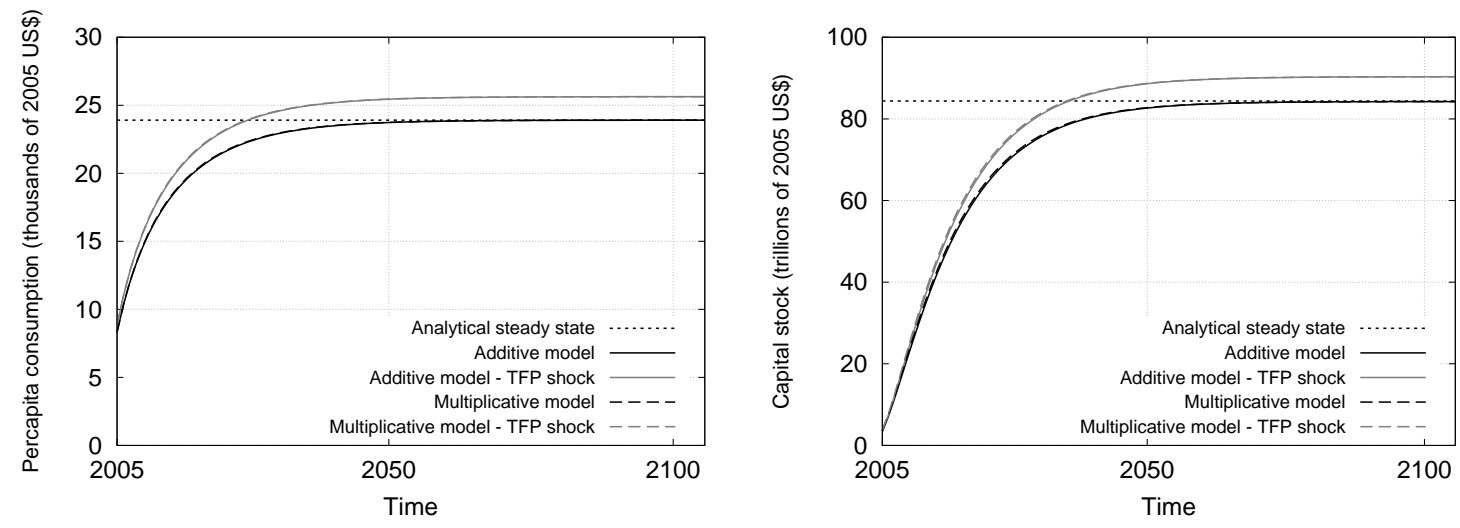

Further evidence about the similarity between the two choice models in the absence of an endogenous catastrophic risk is provided by looking at the response to a TFP shock. Figure 2 displays the optimal transition paths under a $5 \%$ increase in the TFP parameter (A) from $t=0$ onwards (curve labeled "TFP shock"). We find that both the steady state 
values and the transition paths remain almost identical in both models.

\subsection{Optimal climate policy under endogenous catastrophic collapse}

We now consider the case where the planner takes into account the relationship between the stock of pollution and the hazard risk of catastrophic collapse. Optimal paths for consumption $c_{t}$, pollution stock $M_{t}$ and the hazard risk $\mu_{t}$ under the two alternative specifications of the risk profiles and social preferences are reported in Figure 3. We also report BAU trajectories as a reference, which are labeled as "no risk".

In the presence of a collapse risk, consumption in every period is lower than under BAU. However, the magnitude of the decline varies greatly across risk profiles and preferences representations. Starting with the low risk profile, the consumption trajectory under the additive model does not differ significantly from BAU. Similarly, the stock of GHG remains very close to BAU, and the reduction in the hazard risk is negligible. By 2150, the risk of collapse related to the stock of pollution is around $0.01 \%$ per year.

In the framework we consider, a reduction of emissions can be achieved either by using a cleaner but costly technology (i.e. choosing $z_{t}<1$ ), or slowing down the accumulation of capital by increasing consumption. As shown in Figure 4, the optimal solution under additive preferences and a low risk profile only uses the latter option. Since the economy produces with the dirty but efficient technology $\left(z_{t}=1\right)$, slowing down capital accumulation allows the consumption trajectory to remain close to BAU. In the steady state, however, both capital and consumption are lower than their BAU counterpart.

In contrast to the additive model, under multiplicative preferences emissions reductions are drastic even when the planner faces a low risk profile. The dirty technology is used for the first 15 periods, but then $z$ falls below one and the consumption trajectory significantly diverges from BAU. This keeps the steady state stock of emissions below $1200 \mathrm{GtC}$. This threshold, which corresponds to a doubling of GHG concentration compared to the preindustrial level, is therefore never reached with multiplicative preferences.

Turning to the high risk profile, the multiplicative model suggests an immediate use of clean technology, with around $40 \%$ of output being diverted to lower emissions through $z_{t}$. Given this, both the consumption level and the stock of emissions do not grow beyond their 2010 levels. With the additive model there is still no change in technology in the first years, 
Figure 3: Optimal consumption and pollution under endogenous catastrophic risk

(a) Additive model
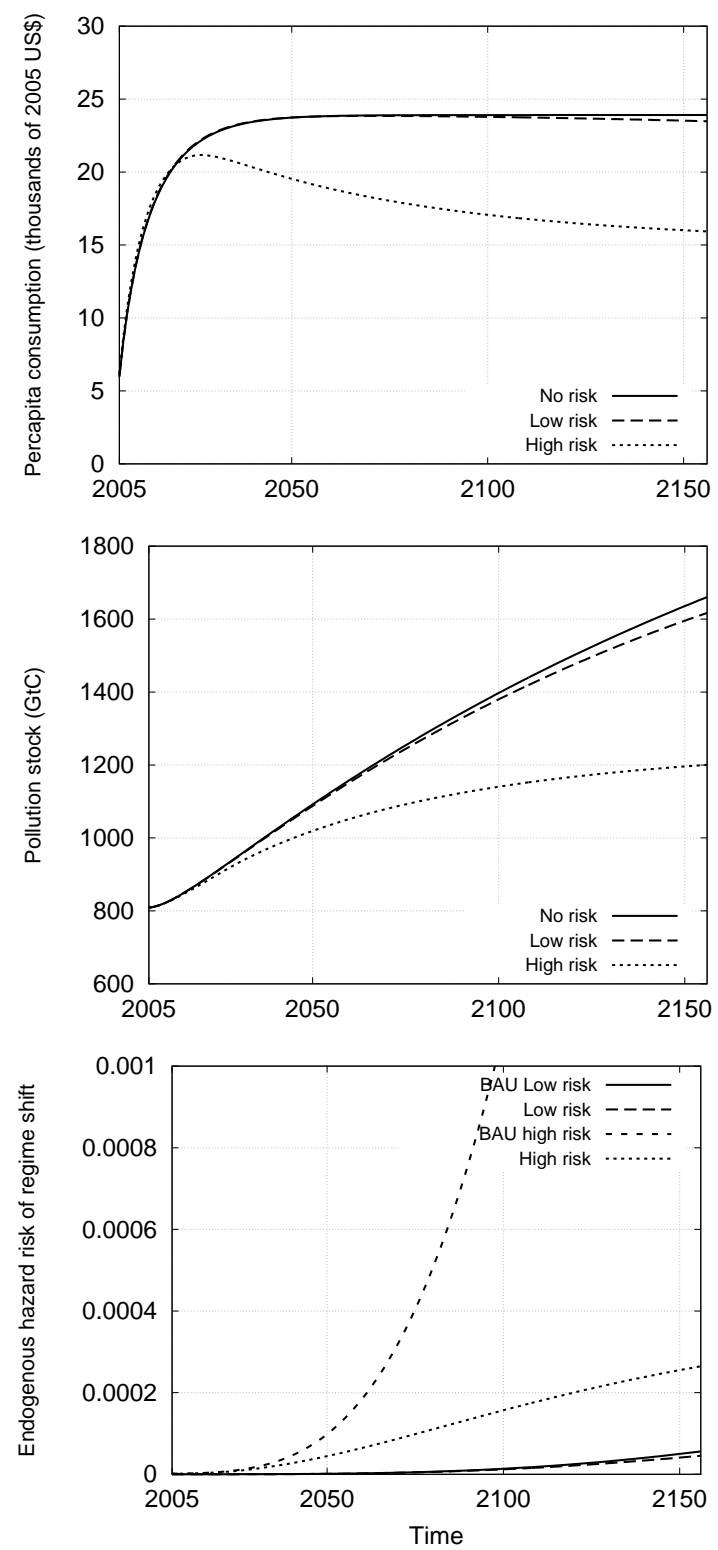

(b) Multiplicative model
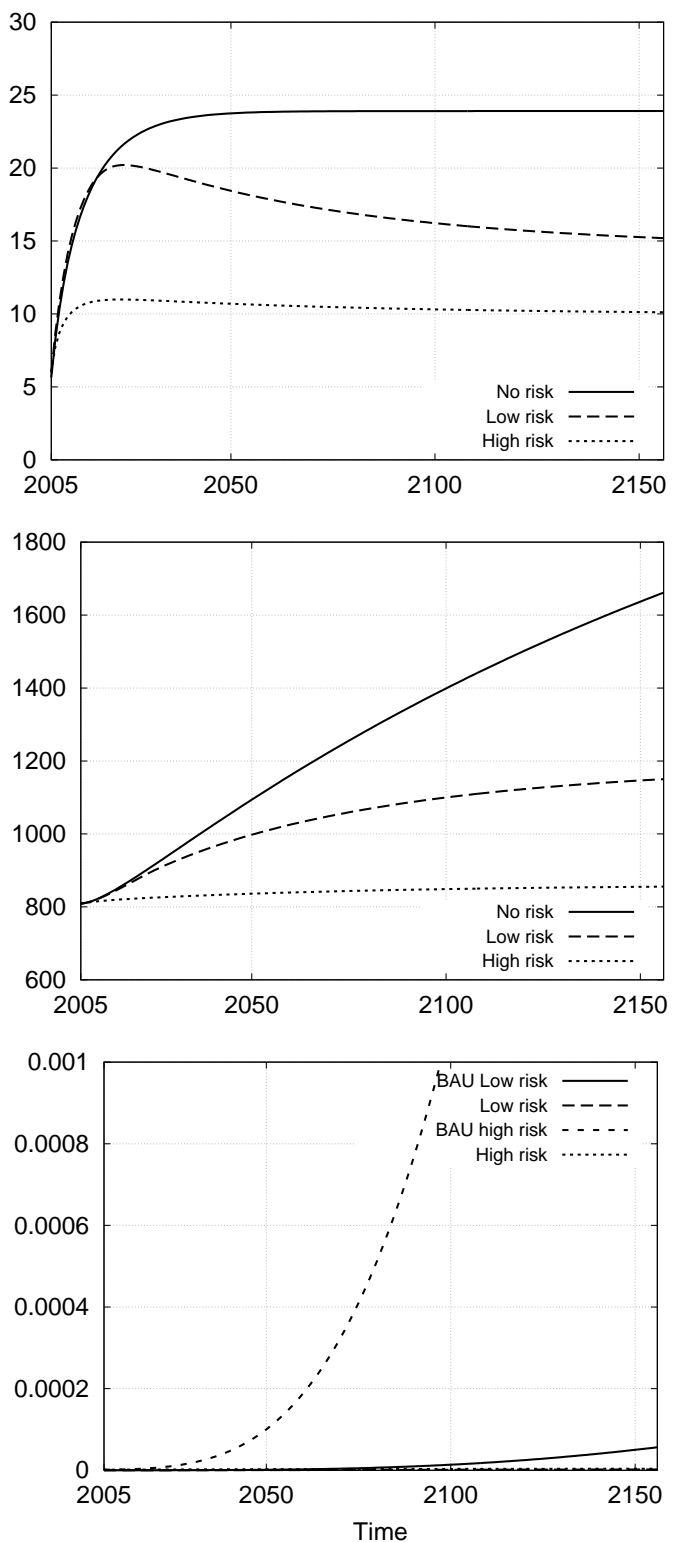

and a much smaller adjustment in the long term. This implies very different level of risks tolerated by the planner. In the multiplicative model the endogenous part of the hazard rate is reduced below the level of the exogenous risk $\left(\mu_{0}=0.001 \%\right)$. With additive preferences, the hazard rate is around $0.02 \%$ by 2100 , which would reduce the survival probability below 
Figure 4: Optimal abatement under endogenous catastrophic risk

(a) Additive model
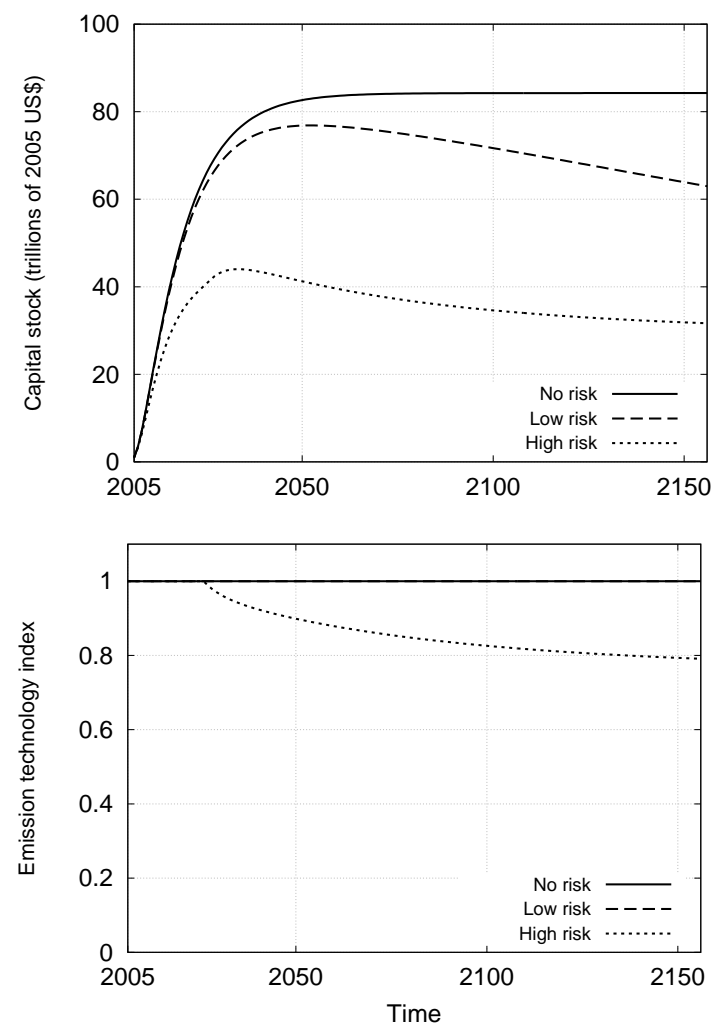

(b) Multiplicative model
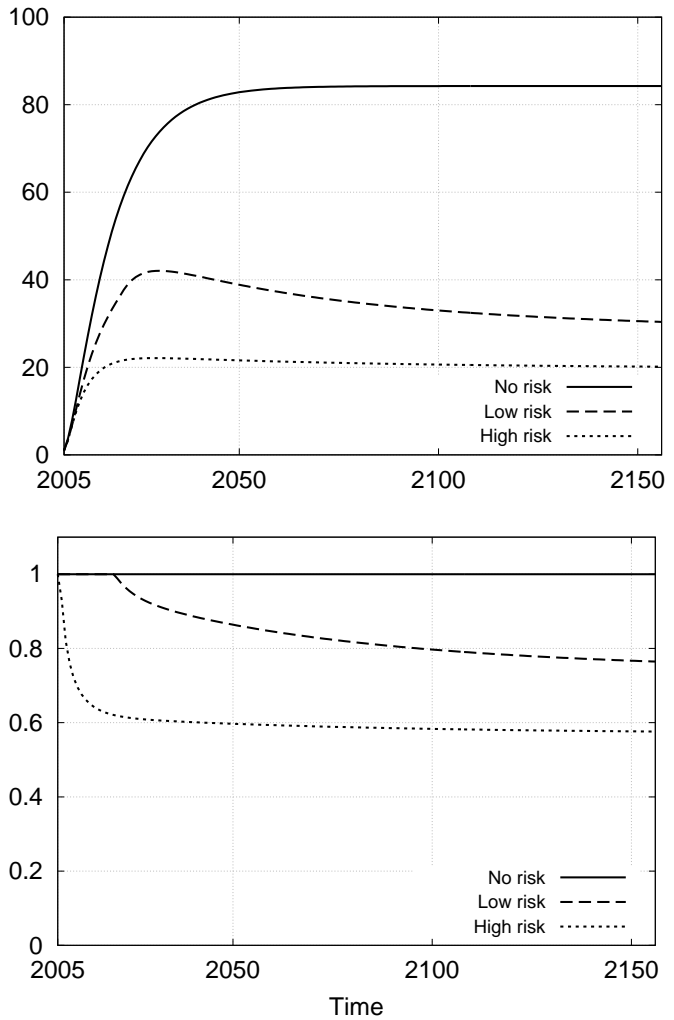

$80 \%$ after 1,000 years.

One may notice that the trajectories obtained with the additive model and high risk is quite close to those derived with the multiplicative model and low risk. Therefore, switching from the additive to the multiplicative model has about the same impact as a 100-fold increase in the hazard risk profile. This suggests that the attention given to the form of social preferences matters as much as the quantification of risks associated with GHG emissions. In particular, in the presence of an unusual catastrophic risk such as climate change, where there are little observations to inform the representation of social preferences, it should at least be recognized that the assumptions underlying the traditional additive model have tremendous consequences for policy recommendations. 


\subsection{The role of post-collapse welfare and time preferences}

Let us now turn to the role of main parameters determining the magnitude of the catastrophe, namely the level of post-collapse consumption $\underline{c}$, and the choice of the discount rate in the additive model (which also determines temporal risk aversion in the multiplicative model).

The choice of $\underline{c}$ does not influence the qualitative nature of our results, but Figure 5 shows that it greatly matters for the magnitude of the policy response. The value for $\underline{c}$ depends on the kind of collapse risk one may think of, and is thus debatable. Our aim here is only to illustrate how this parameter influences our quantitative results. The value used so far $(\underline{c}=1)$ corresponds to an $85 \%$ decrease of consumption. We now consider two additional post-collapse consumption levels: (i) $\underline{c}=0.5$ (more severe poverty); (ii) $\underline{c}=0.01$, a prospect that could be associated with human extinction.

Reducing post-collapse consumption from $\underline{c}=1$ to $\underline{c}=0.5$ has a relatively small impact on optimal paths (compare Figure 3 and Figure 5). Moreover, the change in optimal trajectories is similar under additive and multiplicative preferences, although the differences between models remain (i.e. the stock of emissions is significantly lower with the multiplicative model). Similarly, moving to $\underline{c}=0.01$ involves much more drastic intervention but the qualitative differences between models are preserved.

Quantitatively, we observe that the path derived with additive preferences and a low value of post-collapse consumption, $\underline{c}=0.01$, is close to the one derived with multiplicative preferences and $\underline{c}=1$. This suggests a correspondence between the magnitude of the catastrophic collapse and the choice of the social preference representation, similarly to the above discussion about alternative hazard risk schedules. Moreover, our simulations show that, for both models, $\underline{c}=0.01$ with a low risk profile implies roughly the same equilibrium as $\underline{c}=1$ with a high risk profile.

The second parameter we consider, namely the pure rate of time preferences in the additive model $(\theta)$, is a highly contentious parameter in the economic literature on climate change. As we have shown earlier, it partly determines the difference in how the multiplicative and the additive model respond to an endogenous hazard risk (see Section 4.2). The value used by Nordhaus (2008) of $1.5 \%$, which is based on a revealed preference argu- 
Figure 5: The role of post-collapse consumption level $(\underline{c})$ under endogenous hazard risk

(a) Additive model
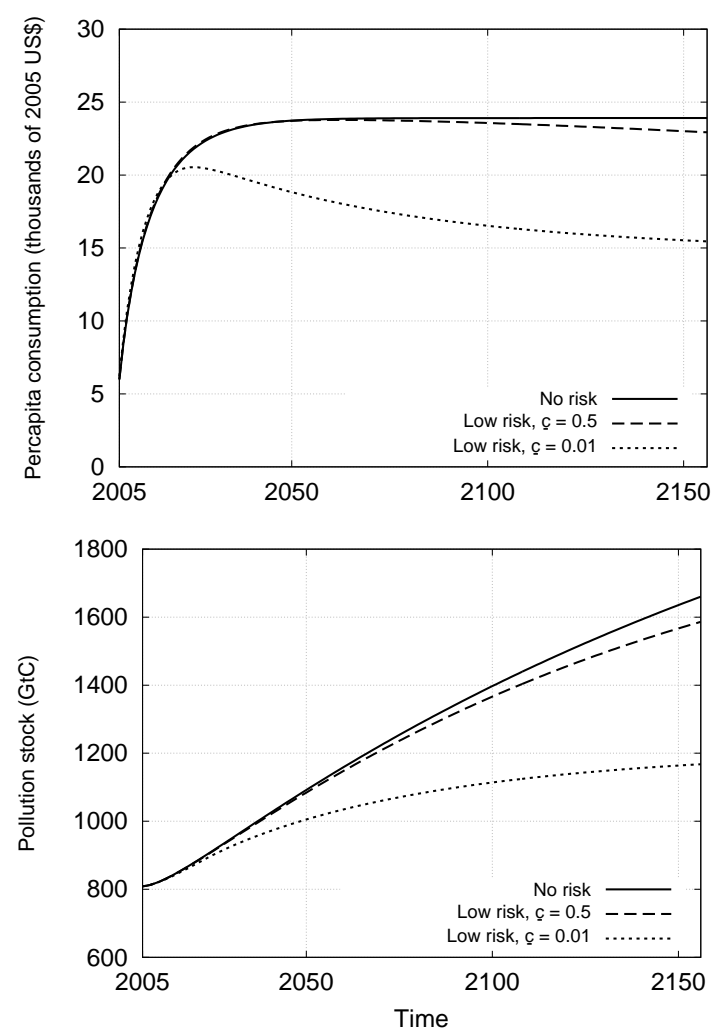

(b) Multiplicative model
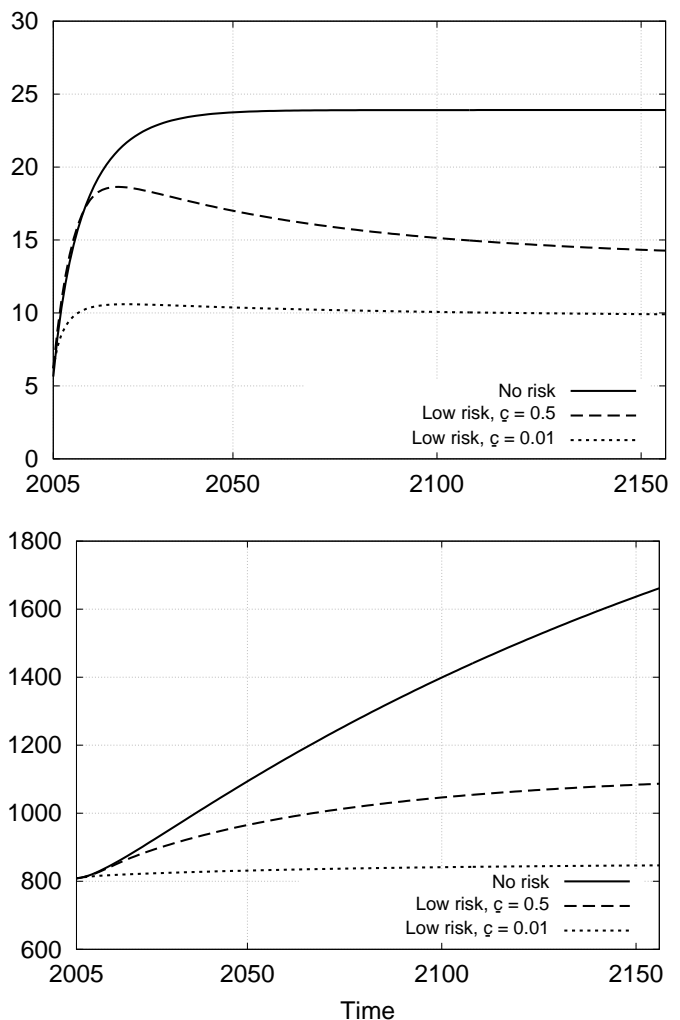

ment using market interest rates, has been criticized by Stern (2007) among others as being unethically high. In the following, we thus use a discount rate of $0.1 \%$ as in Stern (2007).

In order to compare both models, we re-calibrate the parameters so that the two models yield the same BAU steady state interest rate. In particular, the choice of $\theta$ also determines the temporal risk aversion parameter in the multiplicative model $\varepsilon$. Moreover, changing the discount rate also modifies incentives to accumulate capital in the BAU, and we re-calibrate the TFP parameter to remain on the same benchmark path for output and emissions. ${ }^{10}$ Figure 6 compares the additive model with a discount rate of $0.1 \%$, with a multiplicative model re-calibrated to produce the same BAU steady state interest rate. As above, we contrast op-

\footnotetext{
${ }^{10}$ Since our calibration procedure targets the path for output, the BAU consumption path slightly differs from that reported in Figure 3. More important for our analysis, the BAU emissions path and associated hazard risk are however exactly identical.
} 
Figure 6: Comparing additive and multiplicative models under low time preferences $(\theta=$ $0.1 \%)$

(a) Additive model
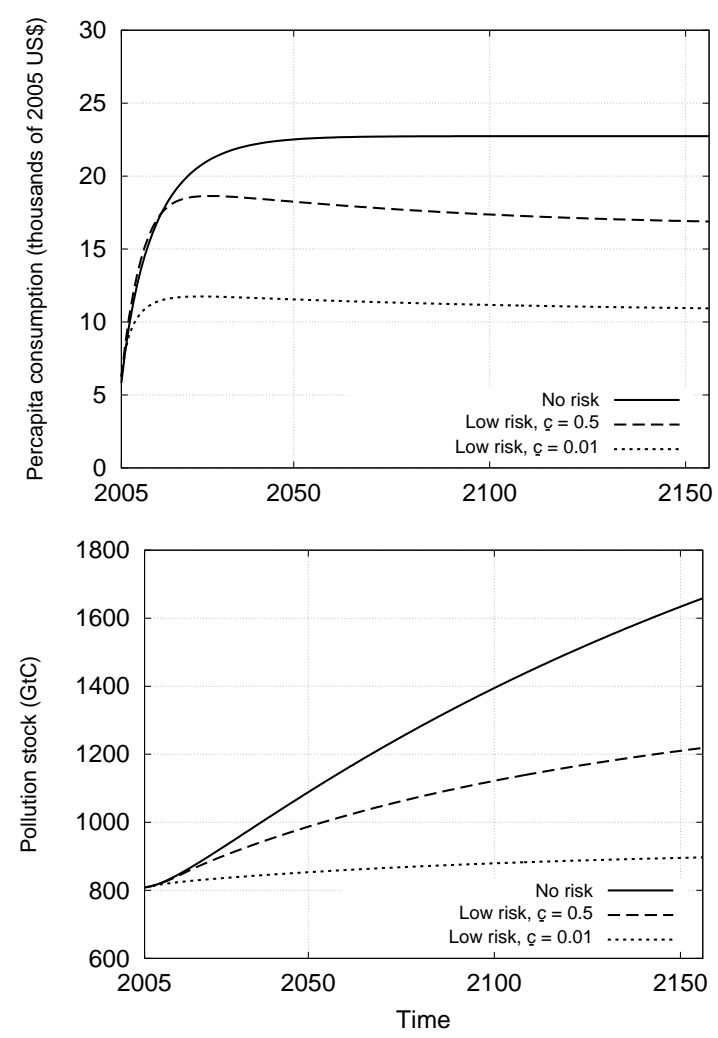

(b) Multiplicative model
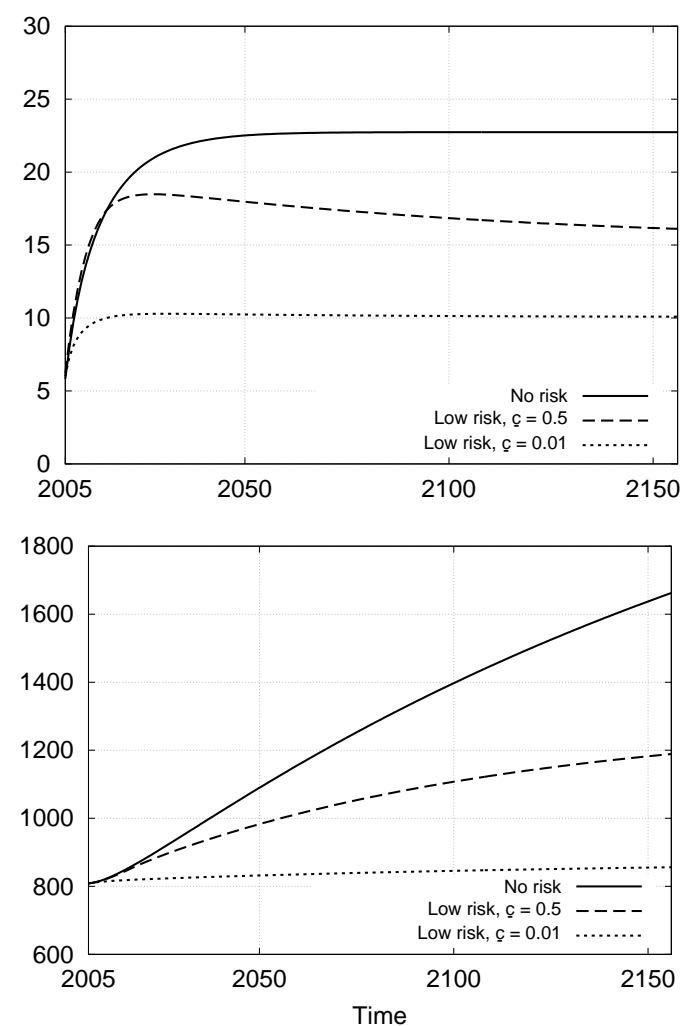

timal paths under the low risk profile and two alternative post-collapse consumption levels, namely $\underline{c}=0.5$ and $\underline{c}=0.01$. We stress that our objective remains to compare how additive and multiplicative models that rationalize the same BAU economy respond to information about a hazard risk of catastrophic collapse.

Under a low value for the pure rate of time preference, here $\theta=0.1 \%$, optimal trajectories for additive and multiplicative preferences are very close. This was expected from Proposition 3: the equations defining the steady state become very similar when the pure rate of time preference approaches zero. The simulations further show that, for the particular parametrization we consider, reducing $\theta$ in the additive model significantly increases the abatement efforts, whereas trajectories for the multiplicative model remain close to those reported in Figure 5. This is so because reducing $\theta$ in the additive model increases the value 
of catastrophic risk reduction, and in the present configuration this effect dominates the discounting effect.

We thus conclude that differences between both models are not irreconcilable, and hinge upon the view of the appropriate pure rate of time preference prevailing in the additive model. Nevertheless, our simulations indicate that it is the paths of the additive model that converge towards those of the multiplicative models rather than the opposite. This therefore reinforces our message that basing policy recommendations solely upon the additive model in conjunction with a more conventional value for the pure rate of time preference $(\theta>1 \%)$ has very strong implications when considering the trade-off between consumption and hazard risk reduction.

\section{Concluding remarks}

Economic studies about individual portfolio choice have long discussed the role of risk aversion. It is now well accepted that individuals may dislike taking risk, and that risk aversion is an element that has to be integrated into studies about the optimal degree of risk taking. Very similarly, one might think that discussions on optimal catastrophic risk prevention should account for risk aversion with respect to intertemporal utility. So far however, the literature on climate change has not done so in a framework able to capture a risk of catastrophic collapse. The reason is that most studies on climate change, and more generally on environmental regime shifts, rely on additively separable preferences, and these preferences are risk neutral with respect to risks on intertemporal utility.

In the current paper, we have considered multiplicative preferences displaying such risk aversion. In the case where catastrophic risks are exogenous and constant, the multiplicative model behaves almost like the standard additive model. However, as soon as we consider endogenous catastrophic risks, the two models radically differ, the multiplicative model advocating a much tighter policy response. We thereby highlight that including risk aversion with respect to intertemporal utility in the analysis has strong implications regarding the appropriate level of action. Loosely speaking, selecting the representation of preferences has the same quantitative implication, in terms of optimal plans, as a large change in the catastrophic risk schedule. Considering alternative choice models may therefore seem 
relevant for the kind of risk associated with climate change.

Our paper takes an original position within the debate on climate policy. Since the report by Stern (2007) the debate has focused on the opposition between a prescriptive approach (holding that the choice of a social objectives should be guided by philosophical arguments), and a descriptive approach (arguing that only welfare functions that are compatible with the observed market interest rates should be considered). This debate has focused on the parameters of the additive social welfare function, rather than on its structure. Without entering into this debate, we show that even if we constrain ourselves to social welfare functions that are compatible with the observed interest rates, assumptions regarding the structure of the social welfare functions are central.

Just like for the choice of the pure rate of time preferences, there is a number of important ethical questions surrounding risk aversion with respect to intertemporal utility. In the simple case of a constant welfare and a risk on the planning horizon, Bommier and Zuber (2008) have showed that such risk aversion implies preferences for catastrophe avoidance in the sense of Keeney (1980) and Fishburn (1984): the society prefers a sure number of death (or existing generations) to a risk on this number having the same expectation. There have been debates about the ethical appeal of preferences for catastrophe avoidance. Some, like Fleurbaey (2010), would actually argue in favor of risk equity, that is the opposite of preferences for catastrophe avoidance, because the social planner may dislike ex-post inequalities. Others, like Keeney (1980), Fishburn (1984), Manski and Tetenov (2007) or Bommier and Zuber (2008), argue that positive preferences for catastrophe avoidance is the most natural assumption. Even if there are divergences in opinion about the degree of preference for catastrophe avoidance, the solution which consist in ruling out this parameter for easier tractability does not seem to be defendable. Our work has illustrated that preferences for catastrophe avoidance is a key ingredient for discussing the mitigation of catastrophic risks, and hence climate change. 


\section{Appendix A Proof of Proposition 1}

Proof of (a).

Using Eq. (10) in the additive case, we obtain:

$$
\frac{\partial W(c)}{\partial c_{T}}=\exp \left(-\int_{0}^{T}\left(\theta+\mu_{t}\right) d t\right) u^{\prime}\left(c_{T}\right)
$$

and

$$
\frac{\partial W(c)}{\partial \mu_{T}}=-\int_{T}^{+\infty} \exp \left(-\int_{0}^{t}\left(\theta+\mu_{\tau}\right) d \tau\right) u\left(c_{t}\right) d t
$$

Hence,

$$
-\left.\frac{d}{d T}\left(\log \frac{\partial W(c)}{\partial c_{T}}\right)\right|_{\dot{c}_{T}=0}=-\frac{-\left(\theta+\mu_{T}\right) \exp \left(-\int_{0}^{T}\left(\theta+\mu_{t}\right) d t\right) u^{\prime}\left(c_{T}\right)}{\exp \left(-\int_{0}^{T}\left(\theta+\mu_{t}\right) d t\right) u^{\prime}\left(c_{T}\right)}=\theta+\mu_{T}
$$

and

$$
-\left(\frac{\partial W(c)}{\partial \mu_{T}}\right) /\left(\frac{\partial W(c)}{\partial c_{T}}\right)=\frac{\int_{T}^{+\infty} \exp \left(-\int_{0}^{t}\left(\theta+\mu_{\tau}\right) d \tau\right) u\left(c_{t}\right) d t}{\exp \left(-\int_{0}^{T}\left(\theta+\mu_{t}\right) d t\right) u^{\prime}\left(c_{T}\right)}=\frac{\int_{T}^{+\infty} \exp \left(-\int_{T}^{t}\left(\theta+\mu_{\tau}\right) d \tau\right) u\left(c_{t}\right) d t}{u^{\prime}\left(c_{T}\right)} .
$$

Proof of (b).

Using Eq. (10) in the multiplicative case, we obtain:

$$
\begin{aligned}
\frac{\partial W(c)}{\partial c_{T}} & =\exp \left(-\int_{0}^{T}\left(\varepsilon u\left(c_{t}\right)+\mu_{t}\right) d t\right) u^{\prime}\left(c_{T}\right)-\varepsilon u^{\prime}\left(c_{T}\right) \int_{T}^{+\infty} \exp \left(-\int_{0}^{t}\left(\varepsilon u\left(c_{\tau}\right)+\mu_{\tau}\right) d \tau\right) u\left(c_{t}\right) d t \\
& =\exp \left(-\int_{0}^{T}\left(\varepsilon u\left(c_{t}\right)+\mu_{t}\right) d t\right) u^{\prime}\left(c_{T}\right)(1-\varepsilon \mathcal{U}(c, T))
\end{aligned}
$$

and

$$
\frac{\partial W(c)}{\partial \mu_{T}}=-\int_{T}^{+\infty} \exp \left(-\int_{0}^{t}\left(\varepsilon u\left(c_{\tau}\right)+\mu_{\tau}\right) d \tau\right) u\left(c_{t}\right) d t=-\exp \left(-\int_{0}^{T}\left(\varepsilon u\left(c_{t}\right)+\mu_{t}\right) d t\right) \mathcal{U}(c, T)
$$


Hence,

$$
\begin{gathered}
-\left.\frac{d}{d T}\left(\log \frac{\partial W(c)}{\partial c_{T}}\right)\right|_{\dot{c}_{T}=0}= \\
-\frac{-\left(\varepsilon u\left(c_{T}\right)+\mu_{T}\right) \exp \left(-\int_{0}^{T}\left(\varepsilon u\left(c_{t}\right)+\mu_{t}\right) d t\right) u^{\prime}\left(c_{T}\right)+\varepsilon u^{\prime}\left(c_{T}\right) \exp \left(-\int_{0}^{T}\left(\varepsilon u\left(c_{t}\right)+\mu_{t}\right) d t\right) u\left(c_{T}\right)}{\exp \left(-\int_{0}^{T}\left(\varepsilon u\left(c_{t}\right)+\mu_{t}\right) d t\right) u^{\prime}\left(c_{T}\right)(1-\varepsilon \mathcal{U}(c, T))} \\
=\frac{\mu_{T}}{1-\varepsilon \mathcal{U}(c, T)}
\end{gathered}
$$

and

$$
-\left(\frac{\partial W(c)}{\partial \mu_{T}}\right) /\left(\frac{\partial W(c)}{\partial c_{T}}\right)=\frac{\exp \left(-\int_{0}^{T}\left(\varepsilon u\left(c_{t}\right)+\mu_{t}\right) d t\right) \mathcal{U}(c, T)}{\exp \left(-\int_{0}^{T}\left(\varepsilon u\left(c_{t}\right)+\mu_{t}\right) d t\right) u^{\prime}\left(c_{T}\right)(1-\varepsilon \mathcal{U}(c, T))}=\frac{\mathcal{U}(c, T)}{u^{\prime}\left(c_{T}\right)(1-\varepsilon \mathcal{U}(c, T))}
$$

\section{Appendix B Optimal dynamics and steady state: BAU problem}

In the case of the additive model, the BAU problem is just the standard Ramsey optimal growth problem (with $\mu_{0}+\theta$ replacing $\theta$ ). So the optimal dynamics and the steady state are well-known.

In the multiplicative case, the BAU problem can be rewritten:

$$
\begin{array}{cl}
\max _{c} & \int_{0}^{+\infty} e^{-\Omega_{t}} u\left(c_{t}\right) d t \\
\text { s.t. } & \dot{k}_{t}=f\left(k_{t}\right)-\delta k_{t}-c_{t} \\
& \dot{\Omega}_{t}=\mu_{0}+\varepsilon u\left(c_{t}\right)
\end{array}
$$

The Hamiltonian of this problem is:

$$
\begin{aligned}
H\left(t, c_{t}, z_{t}, k_{t}, M_{t}, \Omega_{t}\right)= & e^{-\Omega_{t}} u\left(c_{t}\right)+\lambda_{t}\left\{f\left(k_{t}\right)-\delta k_{t}-c_{t}\right\} \\
& +\eta_{t}\left\{\mu_{0}+\varepsilon u\left(c_{t}\right)\right\} .
\end{aligned}
$$


The first order necessary conditions of are:

$$
\begin{aligned}
\frac{\partial H}{\partial c} & =e^{-\Omega_{t}} u^{\prime}\left(c_{t}\right)-\lambda_{t}+\eta_{t} \varepsilon u^{\prime}\left(c_{t}\right)=0 \\
\dot{\lambda}_{t} & =-\frac{\partial H}{\partial k}=-\lambda_{t}\left(f^{\prime}\left(k_{t}\right)-\delta\right) \\
\dot{\eta}_{t} & =-\frac{\partial H}{\partial \Omega}=e^{-\Omega_{t}} u\left(c_{t}\right)
\end{aligned}
$$

Equation (35) implies that $\eta_{t}=\eta(0)+\int_{0}^{t} e^{-\Omega_{\tau}} u\left(c_{\tau}\right) d \tau$. The transversality condition $\lim _{t \rightarrow+\infty} \eta_{t}=0$ yields $\eta(0)=-\int_{0}^{+\infty} e^{-\Omega_{\tau}} u\left(c_{\tau}\right) d \tau$. Hence $\eta_{t}=-\int_{t}^{+\infty} e^{-\Omega_{\tau}} u\left(c_{\tau}\right) d \tau$. Denote $\mathcal{U}_{t}=\int_{t}^{+\infty} e^{-\left(\Omega_{\tau}-\Omega_{t}\right)} u\left(c_{\tau}\right) d \tau$ the future expected utility discounted at period $t$. We obtain

$$
\eta_{t}=-e^{-\Omega_{t}} \mathcal{U}_{t}
$$

so that, using (35),

$$
\dot{\mathcal{U}}_{t}=\dot{\Omega} \mathcal{U}_{t}-u\left(c_{t}\right)
$$

Using Equation (36), Equation (33) can be rewritten:

$$
e^{-\Omega_{t}}\left\{1-\varepsilon \mathcal{U}_{t}\right\} u^{\prime}\left(c_{t}\right)=\lambda_{t}
$$

Differentiating each term in the above equation with respect to time and dividing by the corresponding term in the equation, we obtain:

$$
-\dot{\Omega}_{t}-\frac{\varepsilon \dot{\mathcal{U}}_{t}}{1-\varepsilon \mathcal{U}_{t}}+\dot{c}_{t} \frac{u^{\prime \prime}\left(c_{t}\right)}{u^{\prime}\left(c_{t}\right)}=\frac{\dot{\lambda}_{t}}{\lambda_{t}}
$$

Given Equation (37), we obtain $\dot{\Omega}_{t}+\frac{\varepsilon \dot{\mathcal{U}}_{t}}{1-\varepsilon \mathcal{U}_{t}}=\frac{\left(1-\varepsilon \mathcal{U}_{t}\right) \dot{\Omega}_{t}+\varepsilon\left(\dot{\Omega} \mathcal{U}_{t}-u\left(c_{t}\right)\right)}{1-\varepsilon \mathcal{U}_{t}}=\frac{\dot{\Omega}_{t}-\varepsilon u\left(c_{t}\right)}{1-\varepsilon \mathcal{U}_{t}}=\frac{\mu_{0}}{1-\varepsilon \mathcal{U}_{t}}$. Combining this equality, Equation (34) and the previous equation, we have:

$$
\begin{aligned}
\dot{c}_{t} & =\frac{u^{\prime}\left(c_{t}\right)}{u^{\prime \prime}\left(c_{t}\right)}\left(\frac{\mu_{0}}{1-\varepsilon \mathcal{U}_{t}}+\frac{\dot{\lambda}_{t}}{\lambda_{t}}\right) \\
& =-\frac{u^{\prime}\left(c_{t}\right)}{u^{\prime \prime}\left(c_{t}\right)}\left(f^{\prime}\left(k_{t}\right)-\delta-\frac{\mu_{0}}{1-\varepsilon \mathcal{U}_{t}}\right)
\end{aligned}
$$

In the end, the optimal dynamics for the BAU problem with multiplicative preferences 
are given by the dynamic system:

$$
\begin{aligned}
\dot{c}_{t} & =-\frac{u^{\prime}\left(c_{t}\right)}{u^{\prime \prime}\left(c_{t}\right)}\left(f^{\prime}\left(k_{t}\right)-\delta-\frac{\mu_{0}}{1-\varepsilon \mathcal{U}_{t}}\right) \\
\dot{k}_{t} & =f\left(k_{t}\right)-c_{t} \\
\dot{\mathcal{U}}_{t} & =\dot{\Omega} \mathcal{U}_{t}-u\left(c_{t}\right)=\left(\mu_{0}+\varepsilon u\left(c_{t}\right)\right) \mathcal{U}_{t}-u\left(c_{t}\right)
\end{aligned}
$$

In a steady state, $\dot{\mathcal{U}}_{t}=0$ implies that $\mathcal{U}^{*}=\frac{u\left(c^{*}\right)}{\mu_{0}+\varepsilon u\left(c^{*}\right)}$, so that $\frac{\mu_{0}}{1-\varepsilon \mathcal{U}^{*}}=\mu_{0}+\varepsilon u\left(c^{*}\right)$. Consequently, in steady state, Equations (39)-(40) yield the Equations (18)-(19), which characterize a steady state for an interior solution of Problem (13) in the multiplicative case.

\section{Appendix C Optimal dynamics and steady state: Additive model}

Denote $\Omega_{t}=\int_{0}^{t}\{\mu(\tau)+\theta\} d \tau$ and notice that $\dot{\Omega}_{t}=\mu_{t}+\theta=\mu\left(M_{t}\right)+\theta$. Problem (20) can be rewritten:

$$
\begin{array}{cl}
\max _{c, z} & \int_{0}^{+\infty} e^{-\Omega_{t}} u\left(c_{t}\right) d t \\
\text { s.t. } & \dot{k}_{t}=z_{t} f\left(k_{t}\right)-\delta k_{t}-c_{t} \\
& \dot{M}_{t}=\varphi\left(z_{t}\right) f\left(k_{t}\right)-\psi\left(M_{t}-\bar{M}\right) \\
& \dot{\Omega}_{t}=\mu\left(M_{t}\right)+\theta
\end{array}
$$

The Hamiltonian of this problem is

$$
\begin{aligned}
H\left(t, c_{t}, z_{t}, k_{t}, M_{t}, \Omega_{t}\right)= & e^{-\Omega_{t}} u\left(c_{t}\right)+\lambda_{t}\left\{z_{t} f\left(k_{t}\right)-\delta k_{t}-c_{t}\right\} \\
& +\kappa_{t}\left\{\varphi\left(z_{t}\right) f\left(k_{t}\right)-\psi\left(M_{t}-\bar{M}\right)\right\} \\
& +\eta_{t}\left\{\mu\left(M_{t}\right)+\theta\right\} .
\end{aligned}
$$


The first order necessary conditions are:

$$
\begin{aligned}
\frac{\partial H}{\partial c} & =e^{-\Omega_{t}} u^{\prime}\left(c_{t}\right)-\lambda_{t}=0 \\
\frac{\partial H}{\partial z} & =\lambda_{t} f\left(k_{t}\right)+\kappa_{t} \varphi^{\prime}\left(z_{t}\right) f\left(k_{t}\right)=0 \\
\dot{\lambda}_{t} & =-\frac{\partial H}{\partial k}=-\lambda_{t} z_{t} f^{\prime}\left(k_{t}\right)+\lambda_{t} \delta-\kappa_{t} \varphi\left(z_{t}\right) f^{\prime}\left(k_{t}\right) \\
\dot{\kappa}_{t} & =-\frac{\partial H}{\partial M}=-\mu^{\prime}\left(M_{t}\right) \eta_{t}+\kappa_{t} \psi \\
\dot{\eta}_{t} & =e^{-\Omega_{t}} u\left(c_{t}\right)
\end{aligned}
$$

Equation (43) can be rewritten as:

$$
\kappa_{t}=-\frac{\lambda_{t}}{\varphi^{\prime}\left(z_{t}\right)}
$$

Equation (44) hence becomes:

$$
\dot{\lambda}_{t}=-\left(\left\{z_{t}-\frac{\varphi\left(z_{t}\right)}{\varphi^{\prime}\left(z_{t}\right)}\right\} f^{\prime}\left(k_{t}\right)-\delta\right) \lambda_{t}
$$

We denote $\pi\left(z_{t}\right)=z_{t}-\frac{\varphi\left(z_{t}\right)}{\varphi^{\prime}\left(z_{t}\right)}$. Since $\varphi$ is convex, $\pi$ is increasing.

Equation (42) yields $e^{-\Omega_{t}} u^{\prime}\left(c_{t}\right)=\lambda_{t}$. Differentiating both terms of the equation with respect to time and dividing by the corresponding term of the original equation, we obtain:

$$
\frac{u^{\prime \prime}\left(c_{t}\right)}{u^{\prime}\left(c_{t}\right)} \dot{c}_{t}-\dot{\Omega}_{t}=\frac{u^{\prime \prime}\left(c_{t}\right)}{u^{\prime}\left(c_{t}\right)} \dot{c}_{t}-\theta-\mu\left(M_{t}\right)=\frac{\dot{\lambda}_{t}}{\lambda_{t}}
$$

Using Equation (48), we finally get:

$$
\dot{c}_{t}=-\frac{u^{\prime}\left(c_{t}\right)}{u^{\prime \prime}\left(c_{t}\right)}\left(\pi\left(z_{t}\right) f^{\prime}\left(k_{t}\right)-\delta-\theta-\mu\left(M_{t}\right)\right)
$$

Equation (46) implies that $\eta_{t}=\eta(0)+\int_{0}^{t} e^{-\Omega_{\tau}} u\left(c_{\tau}\right) d \tau$. The transversality condition $\lim _{t \rightarrow+\infty} \eta_{t}=0$ yields $\eta(0)=-\int_{0}^{+\infty} e^{-\Omega_{\tau}} u\left(c_{\tau}\right) d \tau$. Hence $\eta_{t}=-\int_{t}^{+\infty} e^{-\Omega_{\tau}} u\left(c_{\tau}\right) d \tau$, so that:

$$
\eta_{t}=-e^{-\Omega_{t}} \mathcal{U}_{t}
$$


and, by (42),

$$
\eta_{t}=-\frac{\mathcal{U}_{t}}{u^{\prime}\left(c_{t}\right)} \lambda_{t}
$$

Using (47) and (50), Equation (45) becomes:

$$
\dot{\kappa}_{t}=\left(\psi-\mu^{\prime}\left(M_{t}\right) \varphi^{\prime}\left(z_{t}\right) \frac{\mathcal{U}_{t}}{u^{\prime}\left(c_{t}\right)}\right) \kappa_{t}
$$

Differentiating each term in Equation (47) with respect to time and dividing by the corresponding term in (47), we obtain:

$$
\frac{\dot{\kappa}_{t}}{\kappa_{t}}=-\dot{z}_{t} \frac{\varphi^{\prime \prime}\left(z_{t}\right)}{\varphi^{\prime}\left(z_{t}\right)}+\frac{\dot{\lambda}_{t}}{\lambda_{t}}
$$

Hence:

$$
\begin{aligned}
\dot{z}_{t} & =\frac{\varphi^{\prime}\left(z_{t}\right)}{\varphi^{\prime \prime}\left(z_{t}\right)}\left(\frac{\dot{\lambda}_{t}}{\lambda_{t}}-\frac{\dot{\kappa}_{t}}{\kappa_{t}}\right) \\
& =\frac{\varphi^{\prime}\left(z_{t}\right)}{\varphi^{\prime \prime}\left(z_{t}\right)}\left(\mu^{\prime}\left(M_{t}\right) \varphi^{\prime}\left(z_{t}\right) \frac{\mathcal{U}_{t}}{u^{\prime}\left(c_{t}\right)}+\delta-\left\{\psi+\pi\left(z_{t}\right) f^{\prime}\left(k_{t}\right)\right\}\right)
\end{aligned}
$$

Gathering all the results and the equations of motion for $k$ and $M$ yields the following dynamic system:

$$
\begin{aligned}
\dot{c}_{t} & =-\frac{u^{\prime}\left(c_{t}\right)}{u^{\prime \prime}\left(c_{t}\right)}\left(\pi\left(z_{t}\right) f^{\prime}\left(k_{t}\right)-\delta-\rho\left(c_{t}, t\right)\right) \\
\dot{z}_{t} & =\frac{\varphi^{\prime}\left(z_{t}\right)}{\varphi^{\prime \prime}\left(z_{t}\right)}\left(\mu^{\prime}\left(M_{t}\right) \varphi^{\prime}\left(z_{t}\right) \mathcal{V}\left(c_{t}, t\right)+\delta-\left\{\psi+\pi\left(z_{t}\right) f^{\prime}\left(k_{t}\right)\right\}\right) \\
\dot{k}_{t} & =z_{t} f\left(k_{t}\right)-c_{t} \\
\dot{M}_{t} & =\varphi\left(z_{t}\right) f\left(k_{t}\right)-\psi\left(M_{t}-\bar{M}\right)
\end{aligned}
$$

In a steady state, $\mathcal{U}^{*}=\frac{u\left(c^{*}\right)}{\mu\left(M^{*}\right)+\theta}$. Hence Equations (51)-(54) yield Equations (51)-(54), which characterize a steady state for an interior solution of Problem (20) in the additive case. 


\section{Appendix D Optimal dynamics and steady state: Multiplicative model}

Problem (20) can be rewritten:

$$
\begin{array}{cl}
\max _{c, z} & \int_{0}^{+\infty} e^{-\Omega_{t}} u\left(c_{t}\right) d t \\
\text { s.t. } & \dot{k}_{t}=z_{t} f\left(k_{t}\right)-\delta k_{t}-c_{t} \\
& \dot{M}_{t}=\varphi\left(z_{t}\right) f\left(k_{t}\right)-\psi\left(M_{t}-\bar{M}\right) \\
& \dot{\Omega}_{t}=\mu\left(M_{t}\right)+\varepsilon u\left(c_{t}\right)
\end{array}
$$

The Hamiltonian of this problem is

$$
\begin{aligned}
H\left(t, c_{t}, z_{t}, k_{t}, M_{t}, \Omega_{t}\right)= & e^{-\Omega_{t}} u\left(c_{t}\right)+\lambda_{t}\left\{z_{t} f\left(k_{t}\right)-\delta k_{t}-c_{t}\right\} \\
& +\kappa_{t}\left\{\varphi\left(z_{t}\right) f\left(k_{t}\right)-\psi\left(M_{t}-\bar{M}\right)\right\} \\
& +\eta_{t}\left\{\mu\left(M_{t}\right)+\varepsilon u\left(c_{t}\right)\right\} .
\end{aligned}
$$

The first order necessary conditions are:

$$
\begin{aligned}
\frac{\partial H}{\partial c} & =e^{-\Omega_{t}} u^{\prime}\left(c_{t}\right)-\lambda_{t}+\eta_{t} \varepsilon u^{\prime}\left(c_{t}\right)=0 \\
\frac{\partial H}{\partial z} & =\lambda_{t} f\left(k_{t}\right)+\kappa_{t} \varphi^{\prime}\left(z_{t}\right) f\left(k_{t}\right)=0 \\
\dot{\lambda}_{t} & =-\frac{\partial H}{\partial k}=-\lambda_{t} z_{t} f^{\prime}\left(k_{t}\right)+\lambda_{t} \delta-\kappa_{t} \varphi\left(z_{t}\right) f^{\prime}\left(k_{t}\right) \\
\dot{\kappa}_{t} & =-\frac{\partial H}{\partial M}=\kappa_{t} \psi-\eta_{t} \mu^{\prime}\left(M_{t}\right) \\
\dot{\eta}_{t} & =-\frac{\partial H}{\partial \Omega}=e^{-\Omega_{t}} u\left(c_{t}\right)
\end{aligned}
$$

Equation (56) can be rewritten as:

$$
\kappa_{t}=-\frac{\lambda_{t}}{\varphi^{\prime}\left(z_{t}\right)}
$$

Equation (57) hence becomes:

$$
\dot{\lambda}_{t}=-\left(\pi\left(z_{t}\right) f^{\prime}\left(k_{t}\right)-\delta\right) \lambda_{t}
$$


where $\pi$ is defined like in Appendix C.

Equation (59) implies that $\eta_{t}=\eta(0)+\int_{0}^{t} e^{-\Omega_{\tau}} u\left(c_{\tau}\right) d \tau$. The transversality condition $\lim _{t \rightarrow+\infty} \eta_{t}=0$ yields $\eta(0)=-\int_{0}^{+\infty} e^{-\Omega_{\tau}} u\left(c_{\tau}\right) d \tau$. Hence $\eta_{t}=-\int_{t}^{+\infty} e^{-\Omega_{\tau}} u\left(c_{\tau}\right) d \tau$. Denote $\mathcal{U}_{t}=\int_{t}^{+\infty} e^{-\left(\Omega_{\tau}-\Omega_{t}\right)} u\left(c_{\tau}\right) d \tau$ the future expected utility discounted at period $t$. We obtain

$$
\eta_{t}=-e^{-\Omega_{t}} \mathcal{U}_{t}
$$

so that, using (59),

$$
\dot{\mathcal{U}}_{t}=\dot{\Omega} \mathcal{U}_{t}-u\left(c_{t}\right)
$$

Using Equation (62), Equation (55) can be rewritten:

$$
e^{-\Omega_{t}}\left\{1-\varepsilon \mathcal{U}_{t}\right\} u^{\prime}\left(c_{t}\right)=\lambda_{t}
$$

Differentiating each term in the above equation with respect to time and dividing by the corresponding term in the equation, we obtain:

$$
-\dot{\Omega}_{t}-\frac{\varepsilon \dot{\mathcal{U}}_{t}}{1-\varepsilon \mathcal{U}_{t}}+\dot{c}_{t} \frac{u^{\prime \prime}\left(c_{t}\right)}{u^{\prime}\left(c_{t}\right)}=\frac{\dot{\lambda}_{t}}{\lambda_{t}}
$$

Given Equation (63), we obtain $\dot{\Omega}_{t}+\frac{\varepsilon \dot{\mathcal{U}}_{t}}{1-\varepsilon \mathcal{U}_{t}}=\frac{\left(1-\varepsilon \mathcal{U}_{t}\right) \dot{\Omega}_{t}+\varepsilon\left(\dot{\Omega} \mathcal{U}_{t}-u\left(c_{t}\right)\right)}{1-\varepsilon \mathcal{U}_{t}}=\frac{\dot{\Omega}_{t}-\varepsilon u\left(c_{t}\right)}{1-\varepsilon \mathcal{U}_{t}}=\frac{\mu\left(M_{t}\right)}{1-\varepsilon \mathcal{U}_{t}}$. Combining this equality, Equation (61) and the previous equation, we have:

$$
\begin{aligned}
\dot{c}_{t} & =\frac{u^{\prime}\left(c_{t}\right)}{u^{\prime \prime}\left(c_{t}\right)}\left(\frac{\mu\left(M_{t}\right)}{1-\varepsilon \mathcal{U}_{t}}+\frac{\dot{\lambda}_{t}}{\lambda_{t}}\right) \\
& =-\frac{u^{\prime}\left(c_{t}\right)}{u^{\prime \prime}\left(c_{t}\right)}\left(\pi\left(z_{t}\right) f^{\prime}\left(k_{t}\right)-\delta-\frac{\mu\left(M_{t}\right)}{1-\varepsilon \mathcal{U}_{t}}\right)
\end{aligned}
$$

Equations (55) and (62) also imply $\frac{\lambda_{t}}{\eta_{t}}=\left(\frac{e^{-\Omega_{t}}+\varepsilon \eta_{t}}{\eta_{t}}\right) u^{\prime}\left(c_{t}\right)=-\frac{1-\varepsilon \mathcal{U}_{t}}{\mathcal{U}_{t}} u^{\prime}\left(c_{t}\right)$, so that $\frac{\eta_{t}}{\kappa_{t}}=\frac{\mathcal{U}_{t}}{1-\varepsilon \mathcal{U}_{t}} \frac{\varphi^{\prime}\left(z_{t}\right)}{u^{\prime}\left(c_{t}\right)}$. Hence Equation (58) can be rewritten:

$$
\dot{\kappa}_{t}=\left\{\psi-\frac{\mathcal{U}_{t}}{u^{\prime}\left(c_{t}\right)-\varepsilon u^{\prime}\left(c_{t}\right) \mathcal{U}_{t}} \mu^{\prime}\left(M_{t}\right) \varphi^{\prime}\left(z_{t}\right)\right\} \kappa_{t}
$$

Differentiating each term in Equation (56) with respect to time and dividing by the 
corresponding term in (56), we obtain:

$$
\frac{\dot{\kappa}_{t}}{\kappa_{t}}=-\dot{z}_{t} \frac{\varphi^{\prime \prime}\left(z_{t}\right)}{\varphi^{\prime}\left(z_{t}\right)}+\frac{\dot{\lambda}_{t}}{\lambda_{t}}
$$

Hence, by Equations (61) and (65):

$$
\begin{aligned}
\dot{z}_{t} & =\frac{\varphi^{\prime}\left(z_{t}\right)}{\varphi^{\prime \prime}\left(z_{t}\right)}\left(\frac{\dot{\lambda}_{t}}{\lambda_{t}}-\frac{\dot{\kappa}_{t}}{\kappa_{t}}\right) \\
& =\frac{\varphi^{\prime}\left(z_{t}\right)}{\varphi^{\prime \prime}\left(z_{t}\right)}\left(\frac{\mathcal{U}_{t}}{u^{\prime}\left(c_{t}\right)-\varepsilon u^{\prime}\left(c_{t}\right) \mathcal{U}_{t}} \mu^{\prime}\left(M_{t}\right) \varphi^{\prime}\left(z_{t}\right)+\delta-\left\{\psi+\pi\left(z_{t}\right) f^{\prime}\left(k_{t}\right)\right\}\right)
\end{aligned}
$$

Gathering Equations (63), (64), (66), and the equations of motion for $k$ and $M$ yields the dynamic system:

$$
\begin{aligned}
\dot{c}_{t} & =-\frac{u^{\prime}\left(c_{t}\right)}{u^{\prime \prime}\left(c_{t}\right)}\left(\pi\left(z_{t}\right) f^{\prime}\left(k_{t}\right)-\delta-\rho\left(c_{t}, t\right)\right) \\
\dot{z}_{t} & =\frac{\varphi^{\prime}\left(z_{t}\right)}{\varphi^{\prime \prime}\left(z_{t}\right)}\left(\mathcal{V}\left(c_{t}, t\right) \mu^{\prime}\left(M_{t}\right) \varphi^{\prime}\left(z_{t}\right)+\delta-\left\{\psi+\pi\left(z_{t}\right) f^{\prime}\left(k_{t}\right)\right\}\right) \\
\dot{k}_{t} & =z_{t} f\left(k_{t}\right)-c_{t} \\
\dot{M}_{t} & =\varphi\left(z_{t}\right) f\left(k_{t}\right)-\psi\left(M_{t}-\bar{M}\right) \\
\dot{\mathcal{U}}_{t} & =\dot{\Omega} \mathcal{U}_{t}-u\left(c_{t}\right)=\left(\mu\left(M_{t}\right)+\varepsilon u\left(c_{t}\right)\right) \mathcal{U}_{t}-u\left(c_{t}\right)
\end{aligned}
$$

In a steady state, $\dot{\mathcal{U}}_{t}=0$ implies that $\mathcal{U}^{*}=\frac{u\left(c^{*}\right)}{\mu\left(M^{*}\right)+\varepsilon u\left(c^{*}\right)}$, so that $\rho=\frac{\mu\left(M^{*}\right)}{1-\varepsilon \mathcal{U}^{*}}=\mu\left(M^{*}\right)+$ $\varepsilon u\left(c^{*}\right)$ and $\mathcal{V}^{*}=\frac{\mathcal{U}^{*}}{u^{\prime}\left(c^{*}\right)-\varepsilon u^{\prime}\left(c^{*}\right) \mathcal{U}^{*}}=\frac{u\left(c^{*}\right)}{u^{\prime}\left(c^{*}\right) \mu\left(M^{*}\right)}$. Consequently, in steady state, Equations (67)-(70) yield the Equations (29)-(32), which characterize a steady state for an interior solution of Problem (20) in the multiplicative case. 


\section{References}

Ahn, C. M. (1989) "The effect of temporal risk aversion on optimal consumption, the equity premium, and the equilibrium interest rate," Journal of Finance, 44 (5), pp. 1411 - 1420.

Allen, M. R. and D. J. Frame (2007) "Call off the quest," Science, 318, 5850, pp. 582-583.

Alley, R. B., J. Marotzke, W. D. Nordhaus, J. T. Overpeck, D. M. Peteet, R. A. Pielke, R. T. Pierrehumbert, P. B. Rhines, T. F. Stocker, L. D. Talley, and J. M. Wallace (2003) "Abrupt climate change," Science, 299 (5615), pp. 2005 - 2010.

Bommier, A. (2006) "Uncertain lifetime and intertemporal choice: risk aversion as a rationale for time discounting," International Economic Review, 47 (4), pp. 1223 - 1246.

(2012) "Life-cycle preferences revisited," Journal of the European Economic Association, forthcoming.

Bommier, A. and F. LeGrand (2013) "A robust approach to risk aversion." CER-ETH Working Paper 13/172.

Bommier, A. and S. Zuber (2008) "Can preferences for catastrophe avoidance reconcile social discounting with intergenerational equity?" Social Choice and Welfare, 31, pp. $415-434$.

Cai, Y., K. L. Judd, and T. S. Lontzek (2013) "The social cost of abrupt climate change." NBER Working Paper 18704.

Chew, S. H. and L. G. Epstein (1990) "Nonexpected utility preferences in a temporal framework with an application to consumption-savings behaviour," Journal of Economic Theory, 50, pp. 54-81.

Clarke, H. and W. Reed (1994) "Consumption/pollution tradeoffs in an environment vulnerable to pollution-related catastrophic collapse," Journal of Economic Dynamics and Control, 18, pp. $991-1010$.

Cropper, M. (1976) "Regulating activities with catastrophic environmental effects," Journal of Environmental Economics and Management, 3, pp. 1 - 15. 
Dionne, G. and L. Eeckhoudt (1985) "Self-insurance, self-protection and increased risk aversion," Economics Letters, 17, pp. 39-42.

Dirkse, S. P. and M. C. Ferris (1995) "The PATH solver: a non-monontone stabilization scheme for mixed complementarity problems," Optimization Methods and Software, 5, pp. $123-156$.

Epstein, L. G. (1987) "A simple dynamic general equilibrium model," Journal of Economic Theory, 41, pp. 68-95.

Epstein, L. G. and S. E. Zin (1989) "Risk aversion, and the temporal behavior of consumption and asset returns: a theoretical framework," Econometrica, 57, pp. 937-969.

Fishburn, P. (1984) "Equity axioms for public risks," Operation Research, 32, pp. 901 - 908.

Fleurbaey, M. (2010) "Assessing risky social situations," Journal of Political Economy, 188 (4), pp. $649-680$.

Gjerde, J., S. Grepperud, and S. Kverndokk (1999) "Optimal climate policy under the possibility of a catastrophe," Resource and Energy Economics, 21 (3-4), pp. 289 - 317.

Gorman, W. G. (1968) "The structure of utility functions," The Review of Economic Studies, 35 , pp. 367-390.

Hansen, L. P. and T. J. Sargent (1995) "Discounted linear exponential quadratic gaussian control," IEEE Transactions on Automatic Control, 40, 5, pp. 968-971.

Jullien, B., B. Salanié, and F. Salanié (1999) "Should more risk-averse agents exert more effort?" Geneva Papers on Risk and Insurance Theory, 24, pp. 19-28.

Karp, L. and Y. Tsur (2011) "Time perspective and climate change policy," Journal of Environmental Economics and Management, 62, pp. 1 - 14 .

Keeney, R. (1980) "Equity and public risk," Operation Research, 28, pp. 527 - 534.

Keller, K., B. Bolker, and D. Bradford (2004) "Uncertain climate thresholds and optimal economic growth," Journal of Environmental Economics and Management, 48, pp. 723 741. 
Kihlstrom, R. E. and L. J. Mirman (1974) "Risk aversion with many commodities," Journal of Economic Theory, 8 (3), pp. $361-388$.

Koopmans, T. C. (1960) "Stationary ordinal utility and impatience," Econometrica, 28, 2, pp. 287-309.

Kreps, D. M. and E. L. Porteus (1978) "Temporal resolution of uncertainty and dynamic choice theory," Econometrica, 46, 1, pp. 185-200, January.

Kriegler, E., J. W. Hall, H. Held, R. Dawson, and H. J. Schellnhuber (2009) "Imprecise probability assessment of tipping points in the climate system," Proceedings of the National Academy of Sciences, 106, pp. 5041 - 5046.

Lau, M., A. Pahlke, and T. Rutherford (2002) "Approximating infinite-horizon models in a complementarity format: a primer in dynamic general equilibrium analysis," Journal of Economic Dynamics and Control, 26, pp. $577-609$.

Lemoine, D. and C. Traeger (2013) "Watch your step: Optimal policy in a tipping climate," American Economic Journal: Economic Policy, forthcoming.

Lenton, T. M., H. Held, E. Kriegler, J. W. Hall, W. Lucht, S. Rahmstorf, and H. J. Schellnhuber (2008) "Tipping elements in the earth's climate system," Proceedings of the National Academy of Sciences, 105 (6), pp. 1786-1793.

Manski, C. F. and A. Tetenov (2007) "Admissible treatment rules for a risk-averse planner with experimental data on an innovation," Journal of Statistical Planning and Inference, 137, pp. 1998-2010.

Nordhaus, W. D. (2008) A question of Balance: Weighting the options on global warming policies: Yale University Press.

Pye, G. (1973) "Lifetime portfolio selection in continuous time for a multiplicative class of utility functions," American Economic Review, 63, pp. 1013 - 1016.

Roe, G. H. and M. B. Baker (2007) "Why is climate sensitivity so unpredictable?" Science, 318,5850 , pp. 629-632. 
Stern, N. (2007) The Economics of Climate Change: The Stern Review: Cambridge (UK): Cambridge University Press.

Stockey, N. (1998) "Are there limits to growth?" International Economic Review, 39, pp. 1 $-31$.

Tsur, Y. and C. Withagen (2013) "Preparing for catastrophic climate change," Journal of Economics, 110 (3), pp. $225-239$.

Tsur, Y. and A. Zemel (1996) "Accounting for global warming risks: Resource management under event uncertainty," Journal of Economic Dynamics and Control, 20, pp. 1289 1305.

_ (1998) "Pollution control in an uncertain environment," Journal of Economic Dynamics and Control, 22, pp. $967-975$.

van der Ploeg, F. (1993) "A closed-form solution for a model of precautionary saving," Review of Economic Studies, 60 (2), pp. 385 - 395.

Weitzman, M. (2009) "On modeling and interpreting the economics of catastrophic climate change," The Review of Economics and Statistics, 91 (1), pp. 1 - 19.

Yaari, M. E. (1965) "Uncertain lifetime, life insurance, and the theory of the consumer," The Review of Economic Studies, 32, 2, pp. 137-150. 